\title{
Bound states in straight quantum waveguides with combined boundary conditions
}

\author{
J. Dittrich ${ }^{a, b}$ and J. Kř̌iž ${ }^{a, c}$
}

\author{
a) Nuclear Physics Institute, \\ Academy of Sciences of the Czech Republic, 25068 Řež, Czech Republic (mail address) \\ b) Doppler Institute of Mathematical Physics, \\ Faculty of Nuclear Sciences and Physical Engineering, Czech Technical University, \\ Břehová 7, 11519 Prague 1, Czech Republic \\ c) Faculty of Mathematics and Physics, Charles University, \\ V Holešovičkách 2, 18000 Prague 8, Czech Republic \\ dittrich@ujf.cas.cz, kriz@ujf.cas.cz
}

\begin{abstract}
We investigate the discrete spectrum of the Hamiltonian describing a quantum particle living in the two-dimensional straight strip. We impose the combined Dirichlet and Neumann boundary conditions on different parts of the boundary. Several statements on the existence or the absence of the discrete spectrum are proven for two models with combined boundary conditions. Examples of eigenfunctions and eigenvalues are computed numerically.
\end{abstract}

\section{Introduction}

Quantum waveguides with Dirichlet boundary conditions were extensively studied (e.g. 1], 2], [3], [4, [5], [6] and references therein). Their spectral properties essentially depends on the geometry of the waveguide, especially the existence of bound states induced by curvature [1], [2], [3] or

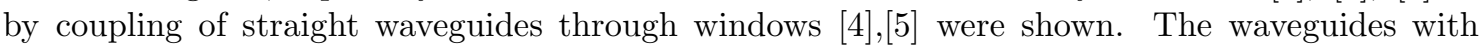
Neumann boundary condition were also investigated in several papers (e.g. [7], [8]). The possible next generalization are waveguides with combined Dirichlet and Neumann boundary conditions on different parts of the boundary. Some very simple combinations of these conditions appear due to the symmetry of special configurations in systems studied e.g. in [4], [5] and [8]. Such "combined" systems might be also of interest directly in nanoscopic physics if interphases modelled by different conditions could be realized. The presence of different boundary conditions also gives rise to nontrivial spectral properties like existence of bound states.

In the present paper, we consider two simple cases of straight planar waveguide of constant width with combined boundary conditions. We show the examples with and without the presence of bound states. The systems we are going to study are sketched on Fig. 1. We consider a Schrödinger particle whose motion is confined to a planar strip of width $d$. For definiteness we assume that it is placed to the upper side of the $x$-axis. On the part of the boundary the Neumann condition is imposed (thin lines in the picture), while on the other part the Dirichlet one holds (thick lines). The length of the overlay of Neumann boundaries is $2 \delta$ and it is placed to both sides of $y$-axis in both cases. We shall denote this configuration space by $\Omega=\mathbb{R} \times(0, d)$ and its particular parts by $\Omega_{I}=(-\infty,-\delta) \times(0, d), \Omega_{I I}=(-\delta, \delta) \times(0, d)$ and $\Omega_{I I I}=(\delta, \infty) \times(0, d)$. As we are going to prove several statements that are valid for more general combination of boundary conditions, let us define several objects. Let there is a finite number of points on the boundary $\partial \Omega$, where boundary condition is changing, which we denote $P_{k}=\left\langle x_{k}, y_{k}\right\rangle, k=1, \ldots, M$. We can choose the numbering so as $y_{k}=d$ for $k=1, \ldots, M^{\prime}$ and $x_{1}<x_{2}<\ldots<x_{M^{\prime}}$ and $y_{k}=0$ for $k=M^{\prime}+1, \ldots, M$ and 
A)

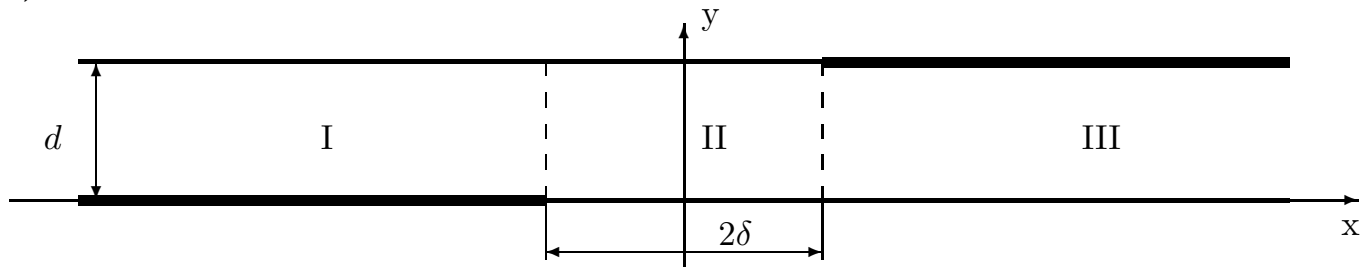

B)

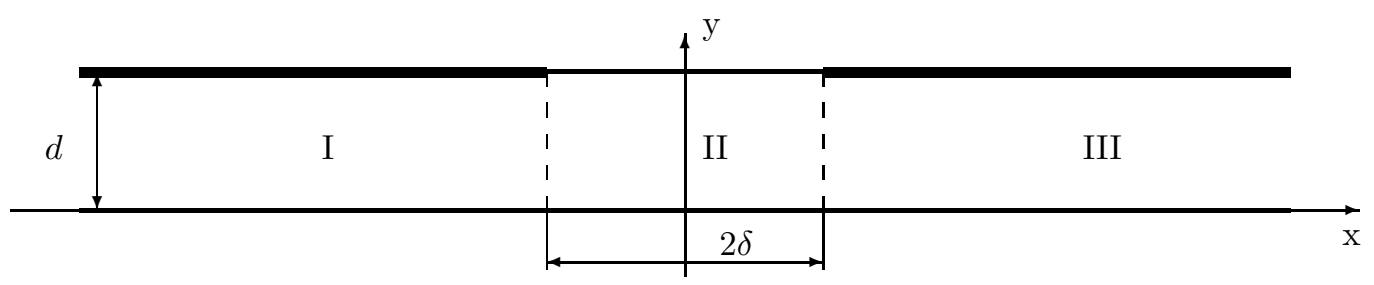

Figure 1: Straight quantum waveguides with combined boundary conditions. The thin lines denote the Neumann boundary condition, the thick lines the Dirichlet one.

$x_{M^{\prime}+1}<x_{M^{\prime}+2}<\ldots<x_{M}$. Let $\partial \Omega=\mathcal{D} \cup \mathcal{N} \cup \bigcup_{k=1}^{M}\left\{P_{k}\right\}$, where $\mathcal{D}$ is a union of finite number of intervals in $\partial \Omega$, where Dirichlet condition is imposed and $\mathcal{N}=(\partial \Omega \backslash \mathcal{D})^{0}$ is similar for Neumann condition. For our examples we have

A)

$$
\begin{aligned}
\mathcal{D} & =\{\langle x, 0\rangle \mid x<-\delta\} \cup\{\langle x, d\rangle \mid x>\delta\} \\
\mathcal{N} & =\{\langle x, 0\rangle \mid x>-\delta\} \cup\{\langle x, d\rangle \mid x<\delta\} \\
P_{1} & =\langle\delta, d\rangle ; P_{2}=\langle-\delta, 0\rangle
\end{aligned}
$$

B)

$$
\begin{aligned}
\mathcal{D} & =\{\langle x, d\rangle \mid(x<-\delta) \vee(x>\delta)\} \\
\mathcal{N} & =\{\langle x, 0\rangle \mid x \in \mathbb{R}\} \cup\{\langle x, d\rangle \mid-\delta<x<\delta\} \\
P_{1} & =\langle-\delta, d\rangle ; P_{2}=\langle\delta, d\rangle
\end{aligned}
$$

In the next section we define the Hamiltonian as Laplace operator with chosen boundary conditions with the help of a quadratic form. We also explicitly give the operator domain which is larger than the Sobolev space $H^{2}(\Omega)$. Due to this fact the proof of its form is a little complicated. In the section III we study the question of bound state existence below the treshold of essential spectrum. The proved results are illustrated in Section IV by numerical calculations. Some technical points are left to Appendices.

\section{The Hamiltonian}

Putting $\hbar^{2} / 2 m=1$, we may identify the particle Hamiltonian with the self-adjoint operator on the Hilbert space $L^{2}(\Omega)$, defined in the following way. Let us define a quadratic form

$$
q_{0}(f, g)=\int_{\Omega} \overline{\nabla f} \cdot \nabla g \mathrm{~d}^{2} x \text { with domain } \mathrm{Q}\left(\mathrm{q}_{0}\right)=\left\{\mathrm{f} \in \mathrm{H}^{1}(\Omega) \mid \mathrm{f} \uparrow \mathcal{D}=0\right\},
$$


where $H^{1}=\left\{f \in L^{2}(\Omega) \mid \nabla f \in L^{2}(\Omega)\right\}$ is the standard Sobolev space and we denote as $f \uparrow \mathcal{D}$ the trace of function $f$ on $\mathcal{D}$. Now $q_{0}$ is obviously densely defined, symmetric and below bounded quadratic form. The form $q_{0}$ is also closed as a direct consequence of Theorem 7.53 in [9].

There is the unique self-adjoint operator associated with this form (see e.g. [10], Theorem 4.6.8). We denote this operator $-\triangle_{D N}^{\Omega}$ and its domain $D(\Omega)$. It is our Hamiltonian. We will show, that this operator acts as the usual Laplace operator with the Dirichlet condition on $\mathcal{D}$ and Neumann condition on $\mathcal{N}$.

Theorem 1 The domain of the operator $-\triangle_{D N}^{\Omega}$ is

$$
\begin{aligned}
D(\Omega) & =\left\{f \in H^{1}(\Omega) \mid-\triangle f \in L^{2}(\Omega), f \uparrow \mathcal{D}=0, \frac{\partial f}{\partial y}\lceil\mathcal{N}=0\},\right. \\
-\triangle_{D N}^{\Omega} f & =-\triangle f \text { for every } f \in D(\Omega) .
\end{aligned}
$$

Proof: First, we know that $D(\Omega) \subset Q\left(q_{0}\right)$. Moreover $f \in D(\Omega)$ if and only if there exists a function $h \in L^{2}(\Omega)$ such that for all $g \in Q\left(q_{0}\right)$ the equality $q_{0}(g, f)=(g, h)_{L^{2}(\Omega)}$ holds. Then $h=-\triangle_{D N}^{\Omega} f$ (see [10], Theorem 4.6.8). Let $g$ be any function from $C_{0}^{\infty}(\Omega)$. Then $g \in Q\left(q_{0}\right)$ and $(\nabla g, \nabla f)_{L^{2}(\Omega)}=(g,-\triangle f)_{L^{2}(\Omega)}$ using only definition of the distributional derivatives. So $-\triangle_{D N}^{\Omega} f=-\triangle f$ for all $f \in D(\Omega)$. We know now

$$
D(\Omega)=\left\{f \in Q\left(q_{0}\right) \mid-\triangle f \in L^{2}(\Omega),\left(\forall g \in Q\left(q_{0}\right)\right)\left((g,-\triangle f)_{L^{2}(\Omega)}=(\nabla g, \nabla f)_{L^{2}(\Omega)}\right)\right\}
$$

Now we prove the implication $f \in D(\Omega) \Rightarrow \frac{\partial f}{\partial y} \uparrow \mathcal{N}=0$. Let $\Omega_{0}$ be an open subset of $\Omega$, such that $P_{k} \notin \bar{\Omega}_{0}, k=1, \ldots, M$. We will show that every $f \in D(\Omega)$ belongs to $H^{2}\left(\Omega_{0}\right)$ for such subdomains. There exist real, positive numbers $\eta_{k}, k=1, \ldots, M$ such that the open balls $B\left(P_{k}, 2 \eta_{k}\right)$ have empty intersections with $\Omega_{0}$. We can choose $\eta_{k}$ so small, that even $\overline{B\left(P_{k}, 2 \eta_{k}\right)} \cap$ $\overline{B\left(P_{k^{\prime}}, 2 \eta_{k^{\prime}}\right)}=\emptyset$ for $k \neq k^{\prime}$. Let we denote

$$
\begin{aligned}
\omega_{0} & =\left(-\infty, x_{1}-\eta_{1}\right) \times(d, 2 d) \\
\vdots & \\
\omega_{M^{\prime}-1} & =\left(x_{M^{\prime}-1}+\eta_{M^{\prime}-1}, x_{M^{\prime}}-\eta_{M^{\prime}}\right) \times(d, 2 d) \\
\omega_{M^{\prime}} & =\left(x_{M^{\prime}}+\eta_{M^{\prime}}, \infty\right) \times(d, 2 d) \\
\omega_{M^{\prime}+1} & =\left(-\infty, x_{M^{\prime}+1}-\eta_{M^{\prime}+1}\right) \times(-d, 0) \\
\vdots & \\
\omega_{M+1} & =\left(x_{M}+\eta_{M}, \infty\right) \times(-d, 0)
\end{aligned}
$$

and let $\tilde{\omega}_{k}$ be the reflection of $\omega_{k}$ to the domain $\Omega$ for $k=0, \ldots, M+1$, i.e. $\tilde{\omega}_{k}=\mathcal{R}_{1}\left(\omega_{k}\right)$ for $k=$ $0, \ldots, M^{\prime}$ and $\tilde{\omega}_{k}=\mathcal{R}_{2}\left(\omega_{k}\right)$ for $k=M^{\prime}+1, \ldots, M+1$, where the bijections $\mathcal{R}_{i}: \mathbb{R}^{2} \rightarrow \mathbb{R}^{2}, i=1,2$ are defined as follows: $\mathcal{R}_{1}(\langle x, y\rangle)=\langle x, 2 d-y\rangle$ and $\mathcal{R}_{2}(\langle x, y\rangle)=\langle x,-y\rangle$. Let $\tilde{\Omega}=\left(\overline{\bigcup_{k=0}^{M+1} \omega_{k} \cup \Omega}\right)^{0}$. In fact, we can say that $\tilde{\Omega}$ is the original domain $\Omega$ with its copy on each side of its boundary, from which we cut the columns $\left[x_{k}-\eta_{k}, x_{k}+\eta_{k}\right] \times[d, 2 d)$, resp. $\left[x_{k}-\eta_{k}, x_{k}+\eta_{k}\right] \times(-d, 0]$, above, resp. below, the point $P_{k}$ depending on which part of $\partial \Omega$ the point $P_{k}$ lies. We construct a function $\tilde{f} \in L^{2}(\tilde{\Omega})$ as follows. For every point $\langle x, y\rangle \in \tilde{\Omega} \backslash \partial \Omega$ we define (so we see that the function will 
be defined almost everywhere in $\tilde{\Omega})$ :

$$
\tilde{f}(x, y)=\left\{\begin{array}{lll}
f(x, y) & \text { for } \quad\langle x, y\rangle \in \Omega & \\
f(x, 2 d-y) & \text { for } \quad\langle x, 2 d-y\rangle \in \Omega, & \langle x, d\rangle \in \mathcal{N} \\
-f(x, 2 d-y) & \text { for } \quad\langle x, 2 d-y\rangle \in \Omega, & \langle x, d\rangle \in \mathcal{D} \\
f(x,-y) & \text { for } \quad\langle x,-y\rangle \in \Omega, & \langle x, 0\rangle \in \mathcal{N} \\
-f(x,-y) & \text { for } \quad\langle x,-y\rangle \in \Omega, & \langle x, 0\rangle \in \mathcal{D} .
\end{array}\right.
$$

Now for any $\varphi \in C_{0}^{\infty}(\tilde{\Omega})$ we can write

$$
\begin{aligned}
& (-\triangle \tilde{f}, \varphi)_{L^{2}(\tilde{\Omega})}=(\tilde{f},-\triangle \varphi)_{L^{2}(\tilde{\Omega})}=(f,-\triangle \varphi)_{L^{2}(\Omega)}+\sum_{k=0}^{M+1}(\tilde{f},-\triangle \varphi)_{L^{2}\left(\omega_{k}\right)}= \\
= & (f,-\triangle \varphi)_{L^{2}(\Omega)} \mp \sum_{k=0}^{M^{\prime}}(-1)^{k} \int_{\tilde{\omega}_{k}} \overline{f(x, y)} \triangle \varphi(x, 2 d-y) \mathrm{d}^{2} x \\
+ & (-1)^{s} \sum_{k=M^{\prime}+1}^{M+1}(-1)^{k} \int_{\tilde{\omega}_{k}} \overline{f(x, y)} \triangle \varphi(x,-y) \mathrm{d}^{2} x=(f,-\triangle \tilde{\varphi})_{L^{2}(\Omega)},
\end{aligned}
$$

where we used the definition of the distributional derivatives and the substitution $y=2 d-y$, resp. $y=-y$. The sign - in $\mp$ is valid for these systems, where Neumann condition is imposed on $\left\{\langle x, d\rangle \mid x \in\left(-\infty, x_{1}\right)\right\}$, the sign + for others. The number $s$ equals 0 or 1 , so as $s+M^{\prime}+1$ is odd for systems, where Neumann condition is imposed on $\left\{\langle x, 0\rangle \mid x \in\left(-\infty, x_{M^{\prime}+1}\right)\right\}$ and even for others. Finally, new function is defined on the domain $\Omega$ as

$$
\tilde{\varphi}(x, y)=\varphi(x, y) \pm \sum_{k=0}^{M^{\prime}}(-1)^{k} \chi_{\tilde{\omega}_{k}}(x, y) \varphi(x, 2 d-y)-(-1)^{s} \sum_{k=M^{\prime}+1}^{M+1}(-1)^{k} \chi_{\tilde{\omega}_{k}}(x, y) \varphi(x,-y),
$$

where $\chi_{\omega}$ is the standard characteristic function of the set $\omega$. Taking into consideration the construction of the domain $\tilde{\Omega}$ and that supp $\varphi \subset \tilde{\Omega}$, we conclude that $\tilde{\varphi} \in C^{\infty}(\bar{\Omega})$ and it has a bounded support. Further, we know that the trace $\tilde{\varphi}\left\lceil\partial \Omega\right.$ equals $\lim _{y \rightarrow d^{-}} \tilde{\varphi}(x, y)$ in the point $\langle x, d\rangle$ and similarly $\lim _{y \rightarrow 0^{+}} \tilde{\varphi}(x, y)$ in the point $\langle x, 0\rangle$ for smooth functions (see the definition of traces, e.g. in 9]). So the traces on $\mathcal{D} \cap \tilde{\Omega}$ are

$$
\begin{aligned}
& \left(\tilde{\varphi}\lceil\partial \Omega)(x, d)=\lim _{y \rightarrow d^{-}} \tilde{\varphi}(x, y)=\lim _{y \rightarrow d^{-}}(\varphi(x, y)-\varphi(x, 2 d-y))=0\right. \\
& \left(\tilde{\varphi}\lceil\partial \Omega)(x, 0)=\lim _{y \rightarrow 0^{+}} \tilde{\varphi}(x, y)=\lim _{y \rightarrow 0^{+}}(\varphi(x, y)-\varphi(x,-y))=0 .\right.
\end{aligned}
$$

In a similar way for parts of boundary $\partial \Omega$ with Neumann condition inside $\tilde{\Omega}$

$$
\begin{aligned}
\left(\frac{\partial \tilde{\varphi}}{\partial y}\lceil\partial \Omega)(x, d)\right. & =\lim _{y \rightarrow d^{-}} \frac{\partial \tilde{\varphi}}{\partial y}(x, y)=\lim _{y \rightarrow d^{-}} \frac{\partial}{\partial y}(\varphi(x, y)+\varphi(x, 2 d-y))=0 \\
\left(\frac{\partial \tilde{\varphi}}{\partial y} \uparrow \partial \Omega\right)(x, 0) & =\lim _{y \rightarrow 0^{+}} \frac{\partial \tilde{\varphi}}{\partial y}(x, y)=\lim _{y \rightarrow 0^{+}} \frac{\partial}{\partial y}(\varphi(x, y)+\varphi(x,-y))=0 .
\end{aligned}
$$

On the rest of the boundary $\partial \Omega$, i.e. $\partial \Omega \backslash \tilde{\Omega}$ both Dirichlet and Neumann conditions are satisfied, what can be seen from the definition of $\tilde{\varphi}$. So it is clear that $\tilde{\varphi} \in H^{2}(\Omega)$ and it satisfies right boundary conditions. It is easy to check, that all such functions belong to $D(\Omega)$, using the Gauss 
Theorem. Because both functions $f$ and $\tilde{\varphi}$ are in $D(\Omega)$, which is a subset of $Q\left(q_{0}\right)$, we can continue the calculation from (2.4).

$$
\begin{aligned}
& (f,-\triangle \tilde{\varphi})_{L^{2}(\Omega)}=(\nabla f, \nabla \tilde{\varphi})_{L^{2}(\Omega)}=(-\triangle f, \tilde{\varphi})_{L^{2}(\Omega)}=(-\triangle f, \varphi)_{L^{2}(\Omega)} \mp \\
\mp & \sum_{k=0}^{M^{\prime}}(-1)^{k} \int_{\omega_{k}} \overline{\triangle f(x, 2 d-y)} \varphi(x, y) \mathrm{d}^{2} x+(-1)^{s} \sum_{k=M^{\prime}+1}^{M+1}(-1)^{k} \int_{\omega_{k}} \overline{\triangle f(x,-y)} \varphi(x, y) \mathrm{d}^{2} x= \\
= & (F, \varphi)_{L^{2}(\tilde{\Omega})},
\end{aligned}
$$

where we used the "reflection" substitution again and $F$ is a function defined by the last formula. Here $F \in L^{2}(\tilde{\Omega})$, because it is the sum of the finite number of $L^{2}$-functions. As we choose the function $\varphi$ arbitrarily, we see that $-\triangle \tilde{f}=F \in L^{2}(\tilde{\Omega})$. Let $\psi \in C^{\infty}\left(\mathbb{R}^{2}\right)$, it is bounded together with its first and second derivatives and let supp $\psi \subset \tilde{\Omega}$. Then $\psi \tilde{f} \in L^{2}\left(\mathbb{R}^{2}\right)$. Using Leibnitz rule and several times a lemma from the section IX.6 in $\left[11\right.$, we conclude that even $-\triangle(\psi \tilde{f}) \in L^{2}\left(\mathbb{R}^{2}\right)$ (Leibnitz rule itself does not give the result unless we know $\left.\nabla \tilde{f} \in L^{2}(\tilde{\Omega})\right)$. We now use this lemma once more and we get the result, that $\psi \tilde{f} \in H^{2}\left(\mathbb{R}^{2}\right)$. We can choose a function $\psi$ so as $\psi\left\lceil\Omega_{0}=1\right.$. It is possible, because $\Omega_{0} \subset \subset \tilde{\Omega}$ and our regions have a simple form at $x \rightarrow \pm \infty$. Let $r_{1}<r_{2}<r_{3}$ be the real positive numbers such that $\left[x_{k}-2 \eta_{k}, x_{k}+2 \eta_{k}\right] \in\left(-r_{1}, r_{1}\right)$ for every $k=1, \ldots, M$ and denote $R_{2}=\left(-r_{2}, r_{2}\right) \times(0, d), R_{3}=\left(-r_{3}, r_{3}\right) \times(-d, 2 d)$. Then using [12], Lemma XIV.2.1 we find a function $\psi_{1} \in C_{0}^{\infty}\left(\tilde{\Omega} \cap R_{3}\right)$, such that $0 \leq \psi_{1}(x) \leq 1$ for all $x \in \tilde{\Omega} \cap R_{3}$ and $\psi_{1}(x)=1$ for $x \in \overline{\Omega_{0} \cap R_{2}}$. This function has compact support in $\Omega$, so its derivatives are bounded. Let now $\gamma \in C_{0}^{\infty}(-d, 2 d)$, such that $\gamma(y)=1$ for $y \in(0, d)$ (it can be constructed according to the same lemma as $\left.\psi_{1}\right)$. Let $\beta \in C^{\infty}(\mathbb{R})$ such that $\beta(x)=1$ for $|x| \geq r_{2}, \beta(x)=0$ on the interval $\left[-r_{1}, r_{1}\right]$ (we can again use the same lemma for construction of $1-\beta$ ). Then $\psi=\psi_{1}(1-\beta)+\gamma \beta$ satisfies all desired properties. Thus $\tilde{f} \psi\left\lceil\Omega_{0}=\tilde{f}\left\lceil\Omega_{0}=f\left\lceil\Omega_{0}\right.\right.\right.$, so $f \in H^{2}\left(\Omega_{0}\right)$.

Now let us take any interval $(a, b)$, such $x_{k} \notin[a, b]$ for $k=1, \ldots, M$. Let $\xi \in C_{0}^{\infty}(a, b)$ be a real function. Because region $(a, b) \times(0, d)$ satisfies all conditions for $\Omega_{0}, f \in H^{2}((a, b) \times(0, d))$. Using Leibnitz rule we can see that $\xi f \in H^{2}((a, b) \times(0, d))$. For any $g \in Q\left(q_{0}\right)$ we have $g \xi \in Q\left(q_{0}\right)$ and

$$
\begin{aligned}
(\nabla g, \nabla(f \xi))_{L^{2}(\Omega)} & =(\nabla g, \xi \nabla f)_{L^{2}(\Omega)}+\left(\frac{\partial g}{\partial x}, f \frac{d \xi}{d x}\right)_{L^{2}(\Omega)}=(\xi \nabla g, \nabla f)_{L^{2}(\Omega)}+ \\
& +\left(g \frac{d \xi}{d x}, \frac{\partial f}{\partial x}\right)_{L^{2}(\Omega)}-\left(g \frac{d \xi}{d x}, \frac{\partial f}{\partial x}\right)_{L^{2}(\Omega)}+\left(\frac{\partial g}{\partial x}, f \frac{d \xi}{d x}\right)_{L^{2}(\Omega)}= \\
& =(g \xi,-\triangle f)_{L^{2}(\Omega)}+\int_{\Omega} \frac{\partial(\overline{g(x, y)} f(x, y))}{\partial x} \frac{d \xi(x)}{d x} \mathrm{~d} x \mathrm{~d} y- \\
& -2\left(g \frac{d \xi}{d x}, \frac{\partial f}{\partial x}\right)_{L^{2}(\Omega)}=(g,-\xi \triangle f)_{L^{2}(\Omega)}-2\left(g, \frac{d \xi}{d x} \cdot \frac{\partial f}{\partial x}\right)_{L^{2}(\Omega)}- \\
& -\left(g, f \frac{d^{2} \xi}{d x^{2}}\right)_{L^{2}(\Omega)}=(g,-\triangle(f \xi))_{L^{2}(\Omega)} .
\end{aligned}
$$

Hence $\xi f \in D(\Omega)$. Using the Gauss Theorem (it can be used for $H^{2}$-functions) we get for any $g \in Q\left(q_{0}\right)$

$$
(g,-\triangle(\xi f))_{L^{2}(\Omega)}=(\nabla g, \nabla(\xi f))_{L^{2}(\Omega)}-\int_{a}^{b}\left(\overline{g(x, d)} \xi(x) \frac{\partial f}{\partial y}(x, d)-\overline{g(x, 0)} \xi(x) \frac{\partial f}{\partial y}(x, 0)\right) \mathrm{d} x
$$

So due to $(2.5)$

$$
\int_{a}^{b}\left(\overline{g(x, d)} \xi(x) \frac{\partial f}{\partial y}(x, d)-\overline{g(x, 0)} \xi(x) \frac{\partial f}{\partial y}(x, 0)\right) \mathrm{d} x=0
$$

for any considered $a, b$ and any $g \in Q\left(q_{0}\right), \xi \in C_{0}^{\infty}(a, b)$. Now we conclude that

$$
\frac{\partial f}{\partial y} \uparrow \mathcal{N}=0 \text { a.e. }
$$


This finishes the second part of proof.

It remains to show that if $f$ satisfies all conditions from (2.2) then $f \in D(\Omega)$ (in the sense of definition (2.3)). Let $\Omega_{0, \varepsilon}=\Omega \backslash \overline{\bigcup_{k=1}^{M} B\left(P_{k}, \varepsilon\right)}, \Omega_{\varepsilon}=\bigcup_{k=1}^{M} B\left(P_{k}, \varepsilon\right) \cap \Omega$. Because we know from the previous part of the proof that $f \in H^{2}\left(\Omega_{0, \varepsilon}\right)$, we can use the Gauss theorem:

$$
\begin{aligned}
& (\nabla g, \nabla f)_{L^{2}(\Omega)}+(g, \Delta f)_{L^{2}(\Omega)}= \\
= & (\nabla g, \nabla f)_{L^{2}\left(\Omega_{0, \varepsilon}\right)}+(g, \Delta f)_{L^{2}\left(\Omega_{0, \varepsilon}\right)}+(\nabla g, \nabla f)_{L^{2}\left(\Omega_{\varepsilon}\right)}+(g, \Delta f)_{L^{2}\left(\Omega_{\varepsilon}\right)}= \\
= & -\sum_{k=1}^{M} \int_{0}^{\pi} \frac{\partial \tilde{f}_{k}(\varepsilon, \varphi)}{\partial r} \overline{\tilde{g}_{k}(\varepsilon, \varphi)} \varepsilon \mathrm{d} \varphi+(\nabla g, \nabla f)_{L^{2}\left(\Omega_{\varepsilon}\right)}+(g, \Delta f)_{L^{2}\left(\Omega_{\varepsilon}\right)},
\end{aligned}
$$

where $\tilde{f}_{k}, \tilde{g}_{k}$ are the transformations of $f, g$ to the polar coordinates in the neighborhood of each $P_{k}$ in the way, that the region $(0, \varepsilon) \times(0, \pi) \subset \Omega, P_{k}$ is the origin of polar coordinates and $\tilde{f}_{k}$ satisfies the Dirichlet condition for $\varphi=0$. We can see that the last two terms in (2.6) go to zero as $\varepsilon \rightarrow 0$, because $\nabla f, \nabla g,-\triangle f, g \in L^{2}(\Omega)$ and the measure of $\Omega_{\varepsilon}$ goes to zero. So we only have to prove, that

$$
\sum_{k=1}^{M} \int_{0}^{\pi} \frac{\partial \tilde{f}_{k}\left(r_{n}, \varphi\right)}{\partial r} \overline{\tilde{g}_{k}\left(r_{n}, \varphi\right)} r_{n} \mathrm{~d} \varphi \rightarrow 0 \text { as } n \rightarrow \infty
$$

for some sequence $\left\{r_{n}\right\}_{n=0}^{\infty}, \lim _{n \rightarrow \infty} r_{n}=0$. We will show that each term in this sum tends to zero. For simplicity we will not write indices in the following text. We will decompose $\tilde{f}$ to the orthonormal transverse basis which respects our boundary conditions.

$$
\tilde{f}(r, \varphi)=\sum_{k=0}^{\infty} \sqrt{\frac{2}{\pi}} F_{k}(r) \sin \frac{2 k+1}{2} \varphi
$$

and in the same way

$$
\tilde{g}(r, \varphi)=\sum_{k=0}^{\infty} \sqrt{\frac{2}{\pi}} G_{k}(r) \sin \frac{2 k+1}{2} \varphi .
$$

Let $R$ be small positive real number, so as $\min _{k \neq k^{\prime}} \operatorname{dist}\left(P_{k}, P_{k^{\prime}}\right)>R$. It is easy to check the following equivalences:

$$
\begin{aligned}
f, g \in H^{1}(\Omega \cap B(P, R)) & \Leftrightarrow \tilde{f}, \tilde{g}, \frac{\partial \tilde{f}}{\partial r}, \frac{\partial \tilde{g}}{\partial r}, \frac{1}{r} \frac{\partial \tilde{f}}{\partial \varphi}, \frac{1}{r} \frac{\partial \tilde{g}}{\partial \varphi} \in L^{2}((0, R) \times(0, \pi), r \mathrm{~d} r \mathrm{~d} \varphi) \\
\triangle f \in L^{2}(\Omega \cap B(P, R)) & \Leftrightarrow\left(\frac{\partial^{2} \tilde{f}}{\partial r^{2}}+\frac{1}{r} \frac{\partial \tilde{f}}{\partial r}+\frac{1}{r^{2}} \frac{\partial^{2} \tilde{f}}{\partial \varphi^{2}}\right) \in L^{2}((0, R) \times(0, \pi), r \mathrm{~d} r \mathrm{~d} \varphi) .
\end{aligned}
$$

Using (2.10) we can decompose $\frac{1}{r} \frac{\partial \tilde{f}}{\partial \varphi}$ to the orthonormal transverse basis

$$
\frac{1}{r} \frac{\partial \tilde{f}}{\partial \varphi}=\sum_{k=0}^{\infty} \sqrt{\frac{2}{\pi}} a_{k}(r) \cos \frac{2 k+1}{2} \varphi .
$$

For almost every $r$ we have

$$
a_{k}(r)=\sqrt{\frac{2}{\pi}} \int_{0}^{\pi} \frac{1}{r} \frac{\partial \tilde{f}}{\partial \varphi}(r, \varphi) \cos \frac{2 k+1}{2} \varphi \mathrm{d} \varphi
$$


and

$$
\begin{aligned}
F_{k}(r) & =\sqrt{\frac{2}{\pi}} \int_{0}^{\pi} \tilde{f}(r, \varphi) \sin \frac{2 k+1}{2} \varphi \mathrm{d} \varphi= \\
& =-\sqrt{\frac{2}{\pi}} \frac{2}{2 k+1} \int_{0}^{\pi} \tilde{f}(r, \varphi) \frac{\partial}{\partial \varphi} \cos \frac{2 k+1}{2} \varphi \mathrm{d} \varphi= \\
& =\sqrt{\frac{2}{\pi}} \frac{2}{2 k+1} \int_{0}^{\pi} \frac{\partial \tilde{f}}{\partial \varphi}(r, \varphi) \cos \frac{2 k+1}{2} \varphi \mathrm{d} \varphi+\sqrt{\frac{2}{\pi}} \frac{2}{2 k+1} \tilde{f}(r, 0)= \\
& =\frac{2}{2 k+1} r a_{k}(r),
\end{aligned}
$$

due to the boundary condition $\tilde{f}(r, 0)=0$. So

$$
a_{k}(r)=\frac{1}{r} \frac{2 k+1}{2} F_{k}(r) .
$$

Now we decompose in the same way $\frac{\partial \tilde{f}}{\partial r}$,

$$
\frac{\partial \tilde{f}}{\partial r}=\sum_{k=0}^{\infty} \sqrt{\frac{2}{\pi}} b_{k}(r) \sin \frac{2 k+1}{2} \varphi .
$$

Let $\left\{\xi_{k, n}\right\}_{n=1}^{\infty}$ be a sequence of $C_{0}^{\infty}(0, \pi)$ functions so as $\lim _{n \rightarrow \infty}\left\|\xi_{k, n}-\sqrt{\frac{2}{\pi}} \sin \frac{2 k+1}{2} \varphi\right\|_{L^{2}(0, \pi)}=0$ for $k=1, \ldots, M$ and let $\omega \in C_{0}^{\infty}(0, R)$. Then using twice the definition of the distributional derivatives, definitions of $F_{k}$ and $b_{k}$ and the fact that $\omega \xi_{k, n} \in C_{0}^{\infty}((0, R) \times(0, \pi))$ we get

$$
\begin{aligned}
& \int_{0}^{R} \frac{d}{d r} F_{k}(r) \omega(r) \mathrm{d} r=-\int_{0}^{R} F_{k}(r) \omega^{\prime}(r) \mathrm{d} r= \\
= & -\int_{0}^{R} \omega^{\prime}(r) \int_{0}^{\pi} \sqrt{\frac{2}{\pi}} \tilde{f}(r, \varphi) \sin \frac{2 k+1}{2} \varphi \mathrm{d} \varphi \mathrm{d} r= \\
= & -\lim _{n \rightarrow \infty} \int_{0}^{R} \int_{0}^{\pi} \omega^{\prime}(r) \xi_{k, n}(\varphi) \tilde{f}(r, \varphi) \mathrm{d} \varphi \mathrm{d} r=\lim _{n \rightarrow \infty} \int_{0}^{R} \int_{0}^{\pi} \omega(r) \xi_{k, n}(\varphi) \frac{\partial \tilde{f}}{\partial r}(r, \varphi) \mathrm{d} \varphi \mathrm{d} r= \\
= & \int_{0}^{R} \omega(r) \int_{0}^{\pi} \sqrt{\frac{2}{\pi}} \frac{\partial}{\partial r} \tilde{f}(r, \varphi) \sin \frac{2 k+1}{2} \varphi \mathrm{d} \varphi \mathrm{d} r=\int_{0}^{R} b_{k}(r) \omega(r) \mathrm{d} r .
\end{aligned}
$$

Because $\omega(r)$ was chosen arbitrarily, we conclude that

$$
b_{k}(r)=\frac{d F_{k}(r)}{d r}=F_{k}^{\prime}(r) .
$$

The same procedure we can apply to $\tilde{g}(r, \varphi)$. Using(2.12) and (2.13) we know, that the series 2.8) and (2.9) can be differentiated by terms. Now we use the similar derivation for $\triangle f$. Let

$$
\left(\frac{\partial^{2} \tilde{f}}{\partial r^{2}}(r, \varphi)+\frac{1}{r} \frac{\partial \tilde{f}}{\partial r}(r, \varphi)+\frac{1}{r^{2}} \frac{\partial^{2} \tilde{f}}{\partial \varphi^{2}}(r, \varphi)\right)=\sqrt{\frac{2}{\pi}} \sum_{k=0}^{\infty} c_{k}(r) \sin \frac{2 k+1}{2} \varphi .
$$


From the first part of the proof we know $\tilde{f}(r,.) \in H^{2}(0, \pi)$ for a.e. $r$ and we can compute

$$
\begin{aligned}
& \int_{0}^{R}\left(F_{k}^{\prime \prime}(r)+\frac{1}{r} F_{k}^{\prime}(r)-\frac{1}{r^{2}}\left(\frac{2 k+1}{2}\right)^{2} F_{k}(r)\right) r \omega(r) \mathrm{d} r=-\int_{0}^{R} r F_{k}^{\prime}(r) \omega^{\prime}(r) \mathrm{d} r- \\
- & \left(\frac{2 k+1}{2}\right)^{2} \int_{0}^{R} \frac{1}{r} F_{k}(r) \omega(r) \mathrm{d} r=\int_{0}^{R}\left(r \omega^{\prime}(r)\right)^{\prime} \int_{0}^{\pi} \sqrt{\frac{2}{\pi}} \tilde{f}(r, \varphi) \sin \frac{2 k+1}{2} \varphi \mathrm{d} \varphi \mathrm{d} r- \\
- & \left(\frac{2 k+1}{2}\right)^{2} \int_{0}^{R} \frac{\omega(r)}{r} \int_{0}^{\pi} \sqrt{\frac{2}{\pi}} \tilde{f}(r, \varphi) \sin \frac{2 k+1}{2} \varphi \mathrm{d} \varphi \mathrm{d} r= \\
= & \lim _{n \rightarrow \infty} \int_{0}^{R}\left(r \omega^{\prime}(r)\right)^{\prime} \int_{0}^{\pi} \tilde{f}(r, \varphi) \xi_{n, k}(\varphi) \mathrm{d} \varphi \mathrm{d} r+ \\
+ & \int_{0}^{R} \frac{\omega(r)}{r} \int_{0}^{\pi} \sqrt{\frac{2}{\pi}} \tilde{f}(r, \varphi) \frac{d^{2} \sin \frac{2 k+1}{2} \varphi}{d \varphi^{2}} \mathrm{~d} \varphi \mathrm{d} r= \\
= & -\lim _{n \rightarrow \infty}^{R} \int_{0}^{R} \omega^{\prime}(r) \xi_{n, k}(\varphi) r \frac{\partial \tilde{f}}{\partial r}(r, \varphi) \mathrm{d} \varphi \mathrm{d} r+ \\
+ & \int_{0}^{R} \frac{\omega(r)}{r} \sqrt{\frac{2}{\pi}}\left(-\frac{2 k+1}{2} \tilde{f}(r, 0)-(-1)^{k} \frac{\partial \tilde{f}}{\partial \varphi}(r, \pi)+\int_{0}^{\pi} \frac{\partial^{2} \tilde{f}}{\partial \varphi^{2}}(r, \varphi) \sin \frac{2 k+1}{2} \varphi \mathrm{d} \varphi\right) \mathrm{d} r= \\
= & \lim _{n \rightarrow \infty} \int_{0}^{R} \int_{0}^{\pi} \omega(r) \xi_{n, k}(\varphi) \frac{\partial}{\partial r}\left(r \frac{\partial \tilde{f}}{\partial r}(r, \varphi)\right) \mathrm{d} \varphi \mathrm{d} r+ \\
+ & \int_{0}^{R} \frac{\omega(r)}{r} \sqrt{\frac{2}{\pi}} \int_{0}^{\pi} \frac{\partial^{2} \tilde{f}}{\partial \varphi^{2}}(r, \varphi) \sin \frac{2 k+1}{2} \varphi \mathrm{d} \varphi \mathrm{d} r= \\
= & \int_{0}^{R} \int_{0}^{\pi} \sqrt{\frac{2}{\pi}} \omega(r) r\left(\frac{\partial^{2} \tilde{f}}{\partial r^{2}}(r, \varphi)+\frac{1}{r} \frac{\partial \tilde{f}}{\partial r}(r, \varphi)+\frac{1}{r^{2}} \frac{\partial^{2} \tilde{f}}{\partial \varphi^{2}}(r, \varphi)\right) \sin \frac{2 k+1}{2} \varphi \mathrm{d} \varphi \mathrm{d} r= \\
= & \int_{0}^{R} c_{k}(r) r \omega(r) \mathrm{d} r,
\end{aligned}
$$

SO

$$
c_{k}(r)=F_{k}^{\prime \prime}(r)+\frac{1}{r} F_{k}^{\prime}(r)-\frac{1}{r^{2}}\left(\frac{2 k+1}{2}\right)^{2} F_{k}(r) \text { for a.e. } r .
$$

Let us denote $\triangle_{p} \tilde{f}(r, \varphi)=\frac{\partial^{2} \tilde{f}}{\partial r^{2}}(r, \varphi)+\frac{1}{r} \frac{\partial \tilde{f}}{\partial r}(r, \varphi)+\frac{1}{r^{2}} \frac{\partial^{2} \tilde{f}}{\partial \varphi^{2}}(r, \varphi)$. Taking into account 2.11) we will solve an equation $\triangle_{p} \tilde{f}(r, \varphi)=h(r, \varphi)$ for any $h \in L^{2}((0, R) \times(0, \pi), r \mathrm{~d} r \mathrm{~d} \varphi)$. We are seeking, of course, only those solutions, for which the function $f(x, y)$ corresponding to the function $\tilde{f}(r, \varphi)$ remains in the set $H^{1}(B(P, R) \cap \Omega)$. If we decompose a function $h$ to the series $h=$ $\sqrt{\frac{2}{\pi}} \sum_{k=0}^{\infty} H_{k}(r) \sin \frac{2 k+1}{2} \varphi$ we get a set of equations:

$$
F_{k}^{\prime \prime}(r)+\frac{1}{r} F_{k}^{\prime}(r)-\frac{1}{r^{2}}\left(\frac{2 k+1}{2}\right)^{2} F_{k}(r)=H_{k}, \quad k=0, \ldots
$$

We denote $\nu=k+1 / 2$. The solutions of these equations are

$$
F_{0}=r^{\frac{1}{2}} \int_{0}^{r} H_{0}(z) z^{\frac{1}{2}} \mathrm{~d} z-r^{-\frac{1}{2}} \int_{0}^{r} H_{0}(z) z^{\frac{3}{2}} \mathrm{~d} z+C_{1}^{(0)} r^{\frac{1}{2}}+C_{2}^{(0)} r^{-\frac{1}{2}}
$$

and for $k>0$

$$
F_{k}=\frac{1}{2 \nu} r^{\nu} \int_{R}^{r} H_{k}(z) z^{-\nu+1} \mathrm{~d} z-\frac{1}{2 \nu} r^{-\nu} \int_{0}^{r} H_{k}(z) z^{\nu+1} \mathrm{~d} z+C_{1}^{(k)} r^{\nu}+C_{2}^{(k)} r^{-\nu} .
$$

We can compute the first derivatives

$$
F_{0}^{\prime}=\frac{1}{2} r^{-\frac{1}{2}} \int_{0}^{r} H_{0}(z) z^{\frac{1}{2}} \mathrm{~d} z+\frac{1}{2} r^{-\frac{3}{2}} \int_{0}^{r} H_{0}(z) z^{\frac{3}{2}} \mathrm{~d} z+\frac{1}{2} C_{1}^{(0)} r^{-\frac{1}{2}}-\frac{1}{2} C_{2}^{(0)} r^{-\frac{3}{2}}
$$


and for $k>0$

$$
F_{k}^{\prime}=\frac{1}{2} r^{\nu-1} \int_{R}^{r} H_{k}(z) z^{-\nu+1} \mathrm{~d} z+\frac{1}{2} r^{-\nu-1} \int_{0}^{r} H_{k}(z) z^{\nu+1} \mathrm{~d} z+\nu C_{1}^{(k)} r^{\nu-1}-\nu C_{2}^{(k)} r^{-\nu-1} .
$$

Because $\tilde{f}, \frac{\partial \tilde{f}}{\partial r} \in L^{2}((0, R) \times(0, \pi), r \mathrm{~d} \varphi \mathrm{d} r)$, the functions $F_{k}$ and $F_{k}^{\prime}$ have to be in the set $L^{2}((0, R), r \mathrm{~d} r)$ for all $k$. Taking the first two terms in (2.16) we get after application of the triangle and Schwarz inequalities

$$
\begin{aligned}
& \left|\frac{1}{2 \nu} r^{\nu} \int_{R}^{r} H_{k}(z) z^{-\nu+1} \mathrm{~d} z-\frac{1}{2 \nu} r^{-\nu} \int_{0}^{r} H_{k}(z) z^{\nu+1} \mathrm{~d} z\right| \leq \\
& \leq \frac{1}{2 \nu}\left(r^{\nu} \sqrt{\int_{r}^{R}\left|H_{k}(z)\right|^{2} z \mathrm{~d} z} \sqrt{\int_{r}^{R} z^{-2 \nu+1} \mathrm{~d} z}+r^{-\nu} \sqrt{\int_{0}^{r}\left|H_{k}(z)\right|^{2} z \mathrm{~d} z} \sqrt{\int_{0}^{r} z^{2 \nu+1} \mathrm{~d} z}\right) \leq \\
& \leq \frac{1}{2 \nu} r \sqrt{\int_{0}^{R}\left|H_{k}(z)\right|^{2} z \mathrm{~d} z}\left(\sqrt{\frac{1-\left(\frac{R}{r}\right)^{-2 \nu+2}}{2 \nu-2}}+\frac{1}{\sqrt{2 \nu+2}}\right) \leq \\
& \leq \frac{r}{\nu \sqrt{2 \nu-2}} \sqrt{\int_{0}^{R}\left|H_{k}(z)\right|^{2} z \mathrm{~d} z}
\end{aligned}
$$

for $0<r<R$. Using similar procedure on (2.15), (2.17) and (2.18) we get the following inequalities holding for every $k=0,1, \ldots$

$$
\begin{array}{r}
\left|F_{k}-C_{1}^{(k)} r^{\nu}-C_{2}^{(k)} r^{-\nu}\right| \leq \frac{r}{\nu \sqrt{|2 \nu-2|}} \sqrt{\int_{0}^{R}\left|H_{k}(z)\right|^{2} z \mathrm{~d} z} \\
\left|F_{k}^{\prime}-\nu C_{1}^{(k)} r^{\nu-1}+\nu C_{2}^{(k)} r^{-\nu-1}\right| \leq \frac{1}{\sqrt{|2 \nu-2|}} \sqrt{\int_{0}^{R}\left|H_{k}(z)\right|^{2} z \mathrm{~d} z .}
\end{array}
$$

We conclude using this estimate that the first three terms in 2.16$)$ belong to $L^{2}((0, R), r \mathrm{~d} r)$ and thus the forth term has to be in this set too. But it is obvious that $r^{-\nu} \notin L^{2}((0, R), r \mathrm{~d} r)$ for $\nu \geq 3 / 2$. Hence $C_{2}^{(k)}=0$ for $k \geq 1$. Applying the same arguments to 2.17 ) we have also $C_{2}^{(0)}=0$. Moreover the condition $\int_{0}^{R} \sum_{k=0}^{\infty}\left|F_{k}^{\prime}(r)\right|^{2} r \mathrm{~d} r<\infty$ must be satisfied. First we suppose $C_{1}^{(k)}=0$ for all $k$. Then using estimate 2.20$)$

$$
\begin{aligned}
\int_{0}^{R} \sum_{k=0}^{\infty}\left|F_{k}^{\prime}(r)\right|^{2} r \mathrm{~d} r & \leq \int_{0}^{R} r \mathrm{~d} r \sum_{k=0}^{\infty} \frac{1}{2|\nu-1|} \int_{0}^{R}\left|H_{k}(z)\right|^{2} z \mathrm{~d} z \leq \\
& \leq\|h\|_{L^{2}((0, R) \times(0, \pi), r \mathrm{~d} \varphi \mathrm{d} r)}^{2} \int_{0}^{R} r \mathrm{~d} r= \\
& =\frac{R^{2}}{2}\|h\|_{L^{2}((0, R) \times(0, \pi), r \mathrm{~d} \varphi \mathrm{d} r)}^{2} .
\end{aligned}
$$

Thus we must choose the constants $C_{1}^{(k)}$ so as

$$
\sum_{k=0}^{\infty} \int_{0}^{R}\left|C_{1}^{(k)}\right|^{2} \nu^{2} r^{2 \nu-2} r \mathrm{~d} r=\sum_{k=0}^{\infty}\left|C_{1}^{(k)}\right|^{2} \frac{\nu}{2} R^{2 \nu}<\infty .
$$

Now we turn our attention to the function $\tilde{g}(r, \varphi)$. Using (2.10) we can write

$$
u(r):=\sum_{k=0}^{\infty} \frac{1}{r^{2}}\left(\frac{2 k+1}{2}\right)^{2}\left|G_{k}(r)\right|^{2} \in L^{1}((0, R), r \mathrm{~d} r) .
$$


Denoting $v(r)=r u(r)$ we get a function $v \in L^{1}(0, R)$. Thus we can state $\liminf _{r \rightarrow 0^{+}}|r v(r)|=0$. Otherwise $|r v(r)| \geq a>0$ for a.e. small $r$, so $|v(r)| \geq a / r$ for some constant $a$ and a.e. sufficiently small $r$, which is a contradiction with the fact that $v \in L^{1}(0, R)$. Hence we can find a sequence $\left\{r_{n}\right\}_{n=1}^{\infty}$ such that $r_{n} \rightarrow 0^{+}$and

$$
\lim _{n \rightarrow \infty}\left|r_{n} v\left(r_{n}\right)\right|=\lim _{n \rightarrow \infty} \sum_{k=0}^{\infty}\left(\frac{2 k+1}{2}\right)^{2}\left|G_{k}\left(r_{n}\right)\right|^{2}=0 .
$$

Now we are ready to return to (2.7). We rewrite the particular terms of this sum using (2.8) and (2.9).

$$
\int_{0}^{\pi} \frac{\partial \tilde{f}_{k}\left(r_{n}, \varphi\right)}{\partial r} \overline{\tilde{g}_{k}\left(r_{n}, \varphi\right)} r_{n} \mathrm{~d} \varphi=\sum_{k=0}^{\infty} r_{n} F_{k}^{\prime}\left(r_{n}\right) \overline{G_{k}\left(r_{n}\right)}
$$

The r.h.s. of this equality can be estimated using (2.20), the triangle inequality and the Schwarz inequality in the space $\ell^{2}$.

$$
\begin{aligned}
\left|\sum_{k=0}^{\infty} r_{n} F_{k}^{\prime}\left(r_{n}\right) \overline{G_{k}\left(r_{n}\right)}\right| & \leq r_{n} \sum_{k=0}^{\infty}\left|G_{k}\left(r_{n}\right)\right|\left(\sqrt{\left.\int_{0}^{R}\left|H_{k}(z)\right|^{2} z \mathrm{~d} z \frac{1}{\sqrt{|2 \nu-2|}}+\nu\left|C_{1}^{(k)}\right| r_{n}^{\nu-1}\right) \leq}\right. \\
& \leq \sqrt{\sum_{k=0}^{\infty}\left|G_{k}\left(r_{n}\right)\right|^{2}}\left(r_{n}\|h\|_{L^{2}((0, R) \times(0, \pi), r \mathrm{~d} \varphi \mathrm{d} r)}+\sqrt{\sum_{k=0}^{\infty} \nu^{2}\left|C_{1}^{(k)}\right|^{2} r_{n}^{2 \nu}}\right)
\end{aligned}
$$

Because of (2.22) it is enough to show, that the last term in brackets in (2.23) is bounded and we will know that the statement (2.7) holds. Due to (2.21) it is sufficient to prove that the inequality $\nu r^{2 \nu}<R^{2 \nu} / 2$ holds for all $\nu=1 / 2,3 / 2, \ldots$ and sufficiently small $r$. Rewriting the inequality to the form

$$
\frac{r}{R}<\left(\frac{1}{2 \nu}\right)^{\frac{1}{2 \nu}}
$$

we will study the function $\phi(x)=\left(\frac{1}{x}\right)^{\frac{1}{x}}$ on the interval $[1, \infty)$. It is obvious that $\phi$ is continuous and strictly positive on this interval. As

$$
\frac{d}{d x} \phi(x)=\phi(x) \frac{\ln (x)-1}{x^{2}}
$$

$\phi$ reaches its global minimum at the point $x=e$. Thus for $r / R<(1 / e)^{1 / e}$ the inequality (2.24) holds for every $\nu$, which completes the proof.

Remark 1 The fact, that the all functions from the domain of the Hamiltonian, which is defined by the quadratic form, satisfy the right boundary condition is supposed to be well-known for domains with reasonable boundaries. But it is also very often supposed, that the operator domain is the subset of the Sobolev space $H^{2}(\Omega)$, which satisfies the boundary conditions. The systems with combined boundary conditions are examples of operators, for which this assertion is not true. The situation is similar to the systems studied in [13]. It was shown there, that for bounded regions in the plane $\mathbb{R}^{2}$ with piecewise $C^{3}$-boundary, which has finite number of angles larger than $\pi$, there exists for each such angle one function, which is not in $H^{2}(\Omega)$ and which belongs to the operator domain with the Dirichlet boundary condition. The operator domain is then a span of the $H^{2}(\Omega)$ space and all these functions, called Guseva functions.

It is easy to check, that similar functions belong to operator domain in our systems too. Let us take the following function, written in polar coordinates with the origin in some $P_{k}$ and $R<d$.

$$
f(r, \varphi)=\xi(r) r^{\frac{1}{2}} \sin \frac{\varphi}{2},
$$


where $\xi(r) \in C^{\infty}(0, \infty), \xi(r)=1$ on $(0, R / 2)$ and $\xi(r)=0$ for $r>R$. This function satisfies the boundary conditions, $\triangle_{p} f \in L^{2}(\Omega)$ and $f \in H^{1}(\Omega)$, thus $f \in D(\Omega)$. But $f \notin H^{2}(\Omega)$. The trace on the part of the boundary $(-R / 2, R / 2)$ is

$$
\frac{1}{r} \frac{\partial f}{\partial \varphi}(r, \varphi)=\left\{\begin{array}{lll}
\frac{1}{2} r^{-1 / 2} & \text { for } & \varphi=0 \\
0 & \text { for } & \varphi=\pi .
\end{array}\right.
$$

So even the trace of the normal derivatives on the boundary is not square integrable. These are the reasons why we cannot immediately use the Gauss Theorem in the proof of Theorem 1.

The interesting open question arises whether all eigenfunctions have Guseva-like behaviour near the points $P_{k}$.

\section{Bound states}

Now we are going to study our specific systems from Fig. 1. First we localize the essential spectra of these systems and make the first estimate on the number of bound states below the essential spectrum treshold using the technique of the Dirichlet-Neumann bracketing (see e.g. [11], Section XIII.15). Then we will continue with the specification of the number of bound states using variational methods.

\section{III.1 Essential spectrum, number of bound states}

Following arguments are the same for both our system, so we do not distinguish between them in this subsection. Cutting the domain $\Omega$ by the additional Neumann or Dirichlet boundaries parallel to the $y$-axis at $x= \pm \delta$, we get new operators $H^{(N)}, H^{(D)}$ defined in the standard way, using the quadratic form. We can decompose these operators $H^{(j)}=H_{t}^{(j)} \oplus H_{c}^{(j)}, j=N, D$, where the "tail" part corresponds to the two halfstrips and the rest to the central part with the Neumann and Dirichlet condition on the vertical boundaries, respectively. Using Dirichlet-Neumann bracketing we have $H_{t}^{(N)} \oplus H_{c}^{(N)} \leq-\triangle_{D N}^{\Omega} \leq H_{t}^{(D)} \oplus H_{c}^{(D)}$ in the sense of quadratic forms (see [11], Section XIII.15).

Now $\sigma_{\text {ess }}\left(H_{t}^{(j)}\right)=\left[\frac{\pi^{2}}{4 d^{2}}, \infty\right), j=N, D$ (we get this result after simple calculation using Example 4.9.6 in [10] and Corollary of the Theorem VIII.33 in 11]). By the minimax principle (see e.g. [11], Section XIII.1) $-\triangle_{D N}^{\Omega}$ has the same infimum of the essential spectrum. To verify that $\sigma_{\text {ess }}\left(-\triangle_{D N}^{\Omega}\right)$ is indeed the whole interval $\left[\frac{\pi^{2}}{4 d^{2}}, \infty\right)$ we can use the same procedure as for $H_{t}^{(j)}$ (see Example 4.9.6 in 10]). Possible isolated eigenvalues of $-\triangle_{D N}^{\Omega}$ are squeezed between those of $H_{c}^{(j)}, j=N, D$. Because the first eigenvalue of $H_{c}^{(N)}$ is zero, $-\triangle_{D N}^{\Omega}$ has an eigenvalue below the essential spectrum treshold provided $H_{c}^{(D)}$ does, which is true if $\delta>d$.

More generally, the number $N_{D}$ of eigenvalues of $H_{c}^{(D)}$ smaller than $\frac{\pi^{2}}{4 d^{2}}$ equals the largest integer number smaller than $\frac{\delta}{d}$, i.e. $N_{D}=-[-\delta / d]-1$, where [.] denotes the entire part. The number of the "Neumann" eigenvalues of $H_{c}^{(N)}$ is $N_{N}=1+N_{D}$. This means that the number of bound states of $-\triangle_{D N}^{\Omega}$ below the essential spectrum treshold satisfies the inequality

$$
-\left[-\frac{\delta}{d}\right]-1 \leq N \leq-\left[-\frac{\delta}{d}\right] .
$$

We see that $-\triangle_{D N}^{\Omega}$ has isolated eigenvalues, at least for $\delta$ large enough. In the same way, one finds that the $m$-th eigenvalue $\mu_{m}$ of $-\triangle_{D N}^{\Omega}$ is estimated by

$$
\left(\frac{m-1}{\lambda}\right)^{2} \leq \frac{\mu_{m}}{\mu} \leq\left(\frac{m}{\lambda}\right)^{2},
$$


where $\lambda=\delta / d$ and $\mu=\inf \left(\sigma_{\text {ess }}\right)=\frac{\pi^{2}}{4 d^{2}}$, and that the critical value $\lambda_{m}=\delta_{m} / d$ at which $m$-th eigenvalue appears satisfies the bounds

$$
m-1 \leq \lambda_{m} \leq m .
$$

To learn more about the dependence of the eigenvalues and the corresponding eigenfunctions on $\lambda$, we have to use a different technique.

\section{III.2 Existence of bound states}

The above existence argument for $\lambda>1$ is a crude one. In fact, there is no lower bound on the length of the overlay of Neumann boundaries for case B). On the other hand we will show that in system A) the discrete spectrum of the Hamiltonian is empty for small $\lambda$, but the ground state appears sooner than $\lambda=1$. We will distinguish our two cases writing $-\triangle_{D N}^{\Omega, A}$, resp. $-\triangle_{D N}^{\Omega, B}$ instead of $-\triangle_{D N}^{\Omega}$ and $Q^{A}\left(q_{0}\right)$, resp. $Q^{B}\left(q_{0}\right)$ instead of $Q\left(q_{0}\right)$.

Theorem 2 The operator $-\triangle_{D N}^{\Omega, B}$ has an isolated eigenvalue in $[0, \mu)$ for any $\delta>0$.

Proof: We slightly modify for the present purpose the variational proof of the Theorem in 四, which comes out from the variational argument of [2]. The transverse ground-state wavefunction at the "tails" of our strip is

$$
\chi(y)=\sqrt{\frac{2}{d}} \cos \sqrt{\mu} y .
$$

For any $\Phi \in Q^{B}\left(q_{0}\right)$ we put

$$
q[\Phi]=q_{0}(\Phi, \Phi)-\mu\|\Phi\|_{L^{2}(\Omega)}^{2} .
$$

Since the essential spectrum of $-\triangle_{D N}^{\Omega, B}$ starts at $\mu$, we have to find a trial function $\Phi$ such that $q[\Phi]<0$, it has to belong to the form domain $Q^{B}\left(q_{0}\right)$ (see e.g. [14, Chapter 4). Thus in particular we can choose $\Phi$ continuous inside $\Omega$, but not necessarily smooth. Notice first that if $\Phi(x, y)=\varphi(x) \chi(y)$, we have

$$
q[\Phi]=\left\|\varphi^{\prime}\right\|_{L^{2}(\mathbb{R})}^{2} .
$$

To make the longitudinal contribution to the kinetic energy small, we use an external scaling. We choose an interval $J=[-b, b]$ for a positive $b>\delta$ and a function $\varphi \in \mathcal{S}(\mathbb{R})$ such that $\varphi(x)=1$ if $x \in J$; then we define the family $\left\{\varphi_{\sigma} \mid \sigma>0\right\}$ by

$$
\varphi_{\sigma}(x)=\left\{\begin{array}{lll}
\varphi(x) & \text { for } & |x| \leq b \\
\varphi( \pm b+\sigma(x \mp b)) & \text { for } & |x| \geq b
\end{array}\right.
$$

Finally, let us choose a real localization function $j \in C_{0}^{\infty}(-\delta, \delta)$ and define

$$
\Phi_{\sigma, \varepsilon}(x, y)=\varphi_{\sigma}(x)\left(\chi(y)+\varepsilon j(x)^{2}\right)
$$

for any $\sigma, \varepsilon>0$. The main point of the construction is that we modify the factorized function we started with in two mutually disjoint regions, outside and inside the rectangle $J \times(0, d)$. Hence the functions $\varphi_{\sigma}^{\prime}$ and $j^{2}$ have disjoint supports. Using this together with the identity

$$
\left\|\varphi_{\sigma}^{\prime}\right\|_{L^{2}(\mathbb{R})}^{2}=\sigma\left\|\varphi^{\prime}\right\|_{L^{2}(\mathbb{R})}^{2},
$$

the explicit form of the function $\chi$ and (3.5), we get after straightforward calculation

$$
q\left[\Phi_{\sigma, \varepsilon}\right]=\sigma\left\|\varphi^{\prime}\right\|_{L^{2}(\mathbb{R})}^{2}-\varepsilon \frac{\pi}{d} \sqrt{\frac{2}{d}}\|j\|_{L^{2}(\mathbb{R})}^{2}+\varepsilon^{2}\left(4 d\left\|j j^{\prime}\right\|_{L^{2}(\mathbb{R})}^{2}-d \mu\left\|j^{2}\right\|_{L^{2}(\mathbb{R})}^{2}\right) .
$$


By construction, the last two terms on the right hand side of (3.9) are independent of $\sigma$. Moreover, the term linear in $\varepsilon$ is negative, so choosing $\varepsilon$ sufficiently small, we can make it dominate over the quadratic one. Finally, we fix this $\varepsilon$ and choose a small enough $\sigma$ to make the right hand side of (3.9) negative.

Now we move to the case A), where the situation is more complicated.

Theorem 3 There exists a real number $\Lambda_{0} \in(0,1)$, such that the discrete spectrum of the operator $-\triangle_{D N}^{\Omega, A}(\lambda)$ is empty for all $\lambda \leq \Lambda_{0}$ and there exists at least one isolated eigenvalue in the spectrum of this operator for all $\lambda>\Lambda_{0}$.

PROOF: $\quad$ Taking into account that $\mu_{1}(\lambda)=\inf _{\varphi \in Q^{A}\left(q_{0}\right),\|\varphi\|=1} q_{0}(\varphi)$ is a nonincreasing continuous function of $\lambda$ (see Appendix A) and the minimax principle [11], it is sufficient to show that there are two real positive numbers $\Lambda_{1}<\Lambda_{2}, \Lambda_{1}, \Lambda_{2} \in(0,1)$, such that

(i) the discrete spectrum is empty for all $\lambda<\Lambda_{1}$,

(ii) there exists at least one isolated eigenvalue in the spectrum for all $\lambda>\Lambda_{2}$.

We know from the previous subsection that there exists a bound state for $\lambda>1$. Let us search for better estimate $\Lambda_{2}<1$ by the similar variational technique as in the proof of the Theorem 2 . We are seeking the trial function $\Phi \in Q^{A}\left(q_{0}\right)$, for which the functional $q$ defined by (3.4) has a negative value. We again choose $\Phi$ continuous inside $\Omega$, but not necessarily smooth and we use the same trick to make the longitudinal contribution to the kinetic energy small. The problem is, that since we have different transverse ground-state wavefunctions at both tails (region I, resp. III on the Fig.1), we cannot construct the "support function" (like $\varphi_{\sigma} \chi$ in the proof of Theorem 2), which longitudinal derivative has disjoint support with the localization function (like $\varepsilon j$ before).

We start with the trial function

$$
\Phi_{\sigma}(x, y)=\varphi_{\sigma}(x) \sin \frac{\pi y}{2 d}+\psi_{\sigma}(x) \cos \frac{\pi y}{2 d}+\eta(x)+\chi(x) \cos \frac{\pi y}{d},
$$

where, similarly to 3.6 )

$$
\begin{aligned}
& \varphi_{\sigma}(x)= \begin{cases}\varphi(x) & \text { for } \quad x \geq-\delta \\
\varphi(-\delta+\sigma(x+\delta)) & \text { for } \quad x \leq-\delta\end{cases} \\
& \psi_{\sigma}(x)= \begin{cases}\psi(x) & \text { for } x \leq \delta \\
\psi(\delta+\sigma(x-\delta)) & \text { for } \quad x \geq \delta,\end{cases}
\end{aligned}
$$

$\varphi, \psi \in \mathcal{S}(\mathbb{R}), \varphi(-\delta)=\psi(\delta)=1 ; \varphi(x)=0$ on $[\delta, \infty), \psi(x)=0$ on $(-\infty,-\delta], \eta, \chi \in C^{\infty}[-\delta, \delta]$ and $\eta(x)=\chi(x)=0$ for $|x| \geq \delta$. The value 1 of $\varphi(-\delta)$ and $\psi(\delta)$ is not important, it can be any constant without the influence on the result. We choose $\varphi(-\delta)=\psi(\delta)$, because we expect the ground state to be symmetric. We shall assume the functions $\varphi, \psi, \eta, \chi$ to be real. Here we can compare this trial function with that one from (3.7). The role of $\varphi_{\sigma}$ from previous case plays here the functions $\varphi_{\sigma}$ and $\psi_{\sigma}$, while the role of localization function plays here $\eta+\chi \cos \frac{\pi y}{d}$. We decompose the functional $q$ to three parts, in each of which one integrates over the region I, resp.II, resp.III

$$
q\left[\Phi_{\sigma}\right]=q_{I}\left[\Phi_{\sigma}\right]+q_{I I}\left[\Phi_{\sigma}\right]+q_{I I I}\left[\Phi_{\sigma}\right]=\frac{d}{2} \sigma\left(\left\|\varphi^{\prime}\right\|_{L^{2}(-\infty,-\delta)}^{2}+\left\|\psi^{\prime}\right\|_{L^{2}(\delta, \infty)}^{2}\right)+q_{I I}\left[\Phi_{\sigma}\right],
$$

where we used identities similar to (3.8). We see that the first term here is always positive, but it can be arbitrarily small, due to parameter $\sigma$, while the second term does not depend on $\sigma$. We 
easily compute

$$
\begin{aligned}
q_{I I}\left[\Phi_{\sigma}\right] & =\int_{-\delta}^{\delta}\left(\frac{d}{2}\left(\varphi^{\prime}(x)^{2}+\psi^{\prime}(x)^{2}+\chi^{\prime}(x)^{2}\right)+d \eta^{\prime}(x)^{2}+\frac{2 d}{\pi} \varphi^{\prime}(x) \psi^{\prime}(x)+\right. \\
& +\frac{4 d}{3 \pi} \chi^{\prime}(x)\left(\psi^{\prime}(x)-\varphi^{\prime}(x)\right)+\frac{4 d}{\pi} \eta^{\prime}(x)\left(\varphi^{\prime}(x)+\psi^{\prime}(x)\right)+\frac{\pi}{d} \chi(x)(\psi(x)-\varphi(x))+ \\
& \left.+\frac{3 \pi^{2}}{8 d} \chi(x)^{2}-\frac{\pi}{d} \varphi(x) \psi(x)-\frac{\pi^{2}}{4 d} \eta(x)^{2}-\frac{\pi}{d} \eta(x)(\psi(x)+\varphi(x))\right) \mathrm{d} x
\end{aligned}
$$

We choose the solution of the Euler equations

$$
\begin{aligned}
d \varphi^{\prime \prime}(x)+\frac{2 d}{\pi} \psi^{\prime \prime}(x)-\frac{4 d}{3 \pi} \chi^{\prime \prime}(x)+\frac{4 d}{\pi} \eta^{\prime \prime}(x)+\frac{\pi}{d}(\psi(x)+\eta(x)+\chi(x)) & =0 \\
d \psi^{\prime \prime}(x)+\frac{2 d}{\pi} \varphi^{\prime \prime}(x)+\frac{4 d}{3 \pi} \chi^{\prime \prime}(x)+\frac{4 d}{\pi} \eta^{\prime \prime}(x)+\frac{\pi}{d}(\varphi(x)+\eta(x)-\chi(x)) & =0 \\
d \chi^{\prime \prime}(x)+\frac{4 d}{3 \pi}\left(\psi^{\prime \prime}(x)-\varphi^{\prime \prime}(x)\right)-\frac{\pi}{d}(\psi(x)-\varphi(x))-\frac{3 \pi^{2}}{4 d} \chi(x) & =0 \\
2 d \eta^{\prime \prime}(x)+\frac{4 d}{\pi}\left(\psi^{\prime \prime}(x)+\varphi^{\prime \prime}(x)\right)+\frac{\pi}{d}(\psi(x)+\varphi(x))+\frac{\pi^{2}}{2 d} \eta(x) & =0
\end{aligned}
$$

to be a trial function. By linear combinations of these equations we can obtain uncoupled second order differential equations for $\varphi-\psi$ and $\varphi+\psi$. Then the solution with boundary condition mentioned above is obtained,

$$
\begin{aligned}
& \chi(x)=\frac{4}{3 \pi}\left(\frac{\sinh \frac{\sqrt{3} \pi x}{2 d}}{\sinh \frac{\sqrt{3} \pi \delta}{2 d}}-\frac{\sinh \frac{\pi x}{d} \sqrt{3 \frac{3 \pi-8}{9 \pi^{2}-18 \pi-32}}}{\sinh \frac{\pi \delta}{d} \sqrt{3 \frac{3 \pi-8}{9 \pi^{2}-18 \pi-32}}}\right) \\
& \varphi(x)=\frac{1}{2}\left(\frac{\cosh \frac{\pi x}{d} \sqrt{\frac{4-\pi}{\pi^{2}+2 \pi-16}}}{\cosh \frac{\pi \delta}{d} \sqrt{\frac{4-\pi}{\pi^{2}+2 \pi-16}}}-\frac{\sinh \frac{\pi x}{d} \sqrt{3 \frac{3 \pi-8}{9 \pi^{2}-18 \pi-32}}}{\sinh \frac{\pi \delta}{d} \sqrt{3 \frac{3 \pi-8}{9 \pi^{2}-18 \pi-32}}}\right) \\
& \psi(x)=\frac{1}{2}\left(\frac{\cosh \frac{\pi x}{d} \sqrt{\frac{4-\pi}{\pi^{2}+2 \pi-16}}}{\cosh \frac{\pi \delta}{d} \sqrt{\frac{4-\pi}{\pi^{2}+2 \pi-16}}}+\frac{\sinh \frac{\pi x}{d} \sqrt{3 \frac{3 \pi-8}{9 \pi^{2}-18 \pi-32}}}{\sinh \frac{\pi \delta}{d} \sqrt{3 \frac{3 \pi-8}{9 \pi^{2}-18 \pi-32}}}\right) \\
& \eta(x)=\frac{2}{\pi}\left(\frac{\cos \frac{\pi x}{2 d}}{\cos \frac{\pi \delta}{2 d}}-\frac{\cosh \frac{\pi x}{d} \sqrt{\frac{4-\pi}{\pi^{2}+2 \pi-16}}}{\cosh \frac{\pi \delta}{d} \sqrt{\frac{4-\pi}{\pi^{2}+2 \pi-16}}}\right) .
\end{aligned}
$$

As the quadratic form of the derivatives in (3.11) is positive definite this trial function is a good candidate for a minimum of the functional (3.11). Now we substitute (3.12) to (3.11) and after a tedious but straightforward calculation we obtain

$$
\begin{aligned}
q_{I I}\left[\Phi_{\sigma}\right] & =\frac{\sqrt{(4-\pi)\left(\pi^{2}+2 \pi-16\right)}}{2 \pi} \tanh \frac{\pi \delta}{d} \sqrt{\frac{4-\pi}{\pi^{2}+2 \pi-16}}+\frac{8}{3 \sqrt{3} \pi} \operatorname{coth} \frac{\sqrt{3} \pi \delta}{2 d}+ \\
& +\frac{\sqrt{(3 \pi-8)\left(9 \pi^{2}-18 \pi-32\right)}}{6 \sqrt{3} \pi} \operatorname{coth} \frac{\pi \delta}{d} \sqrt{3 \frac{3 \pi-8}{9 \pi^{2}-18 \pi-32}}-\frac{4}{\pi} \tan \frac{\pi \delta}{2 d} .
\end{aligned}
$$

Now we can understand $q_{I I}$ like a function of a variable $\delta$. We see, that $q_{I I}$ is a continuous function on the interval $(0, d), \lim _{\delta \rightarrow 0^{+}} q_{I I}(\delta)=+\infty$ and $\lim _{\delta \rightarrow d^{-}} q_{I I}(\delta)=-\infty$. So there must exist a point 
$\delta_{0} \in(0, d)$ and corresponding number $\Lambda_{2}=\delta_{0} / d$, such that $q_{I I}(\delta)<0$ for $\delta \in\left(\delta_{0}, d\right)$. Thus we can find for every $\delta$ from this interval a number $\sigma$ small enough to have $q\left[\Phi_{\sigma}\right]<0$, which finishes the proof of the existence of $\Lambda_{2}$.

Now we are going to prove, that the discrete spectrum of the operator $-\triangle_{D N}^{\Omega, A}(\lambda)$ is empty for all $\lambda \leq \Lambda_{1}$. It will be shown if we demonstrate that the functional $q[\Phi] \geq 0$ for all $\Phi$ from a suitable dense (in $H^{1}(\Omega)$ norm) set in $Q^{A}\left(q_{0}\right)$, say

$$
\mathcal{Q}(\Omega)=\left\{\psi \in H^{1}(\Omega) \cap C(\bar{\Omega}) \mid \psi \in C^{2}\left(\Omega_{j}\right), j=I, I I, I I I, \psi \uparrow \mathcal{D}=0\right\},
$$

where $C(\bar{\Omega})$ is just the set of functions continuous in the closure of $\Omega$. It can be proven that this set is really dense in $Q^{A}\left(q_{0}\right)$ (See Appendix B). We again decompose $q$ into three parts $q[\Phi]=q_{I}[\Phi]+q_{I I}[\Phi]+q_{I I I}[\Phi]$. The "tail" parts of $\Phi \in \mathcal{Q}(\Omega)$ we expand to the series

$$
\Phi(x, y)=\sqrt{\frac{2}{d}} \sum_{k=0}^{\infty} a_{k}(x) \sin \frac{2 k+1}{2 d} \pi y
$$

in the region $\Omega_{I}$ and

$$
\Phi(x, y)=\sqrt{\frac{2}{d}} \sum_{k=0}^{\infty} b_{k}(x) \cos \frac{2 k+1}{2 d} \pi y
$$

in the region $\Omega_{I I I}$. Using the same procedure like in the proof of the Theorem 1, we know that these series can be differentiated by terms. Hence

$$
\begin{aligned}
q_{I}[\Phi] & =\sum_{k=0}^{\infty} \int_{-\infty}^{-\delta}\left(\left|a_{k}^{\prime}(x)\right|^{2}+\frac{\pi^{2}}{d^{2}}\left(k^{2}+k\right)\left|a_{k}(x)\right|^{2}\right) \mathrm{d} x \\
q_{I I I}[\Phi] & =\sum_{k=0}^{\infty} \int_{\delta}^{\infty}\left(\left|b_{k}^{\prime}(x)\right|^{2}+\frac{\pi^{2}}{d^{2}}\left(k^{2}+k\right)\left|b_{k}(x)\right|^{2}\right) \mathrm{d} x
\end{aligned}
$$

and we see that $q_{I}[\Phi] \geq 0$ and $q_{I I I}[\Phi] \geq 0$ for all $\Phi \in \mathcal{Q}(\Omega)$. As we have seen above (similarly to (3.8)) the contributions from the terms $\left|a_{0}^{\prime}(x)\right|^{2}$ and $\left|b_{0}^{\prime}(x)\right|^{2}$ can be arbitrarily small. We minimalize the rest of the functional using Euler equations, the general boundary condition fixing the function values at $x=\delta$, resp. $x=-\delta$, and the square integrability. As $\Phi \in C(\bar{\Omega})$ the Fourier coefficients $a_{k}$, resp. $b_{k}$, are continuous in $(-\infty,-\delta]$, resp. $[\delta, \infty)$, and $a_{k}(-\delta)$, resp. $b_{k}(-\delta)$, are those of $\Phi(-\delta, \cdot)$, resp. $\Phi(\delta, \cdot)$. The solution of the Euler equations will be really the absolute minimum of $q_{I}+q_{I I I}$. It is easy to see, that for all $\Phi$ in the set $\mathcal{Q}(\Omega), q_{I}[\Phi]+q_{I I I}[\Phi]=$ $q_{I}\left[\Phi_{0}\right]+q_{I I I}\left[\Phi_{0}\right]+q_{I}\left[\Phi-\Phi_{0}\right]+q_{I I I}\left[\Phi-\Phi_{0}\right]$, where $\Phi_{0}$ is given by Euler equations and we have seen above that $q_{I}\left[\Phi-\Phi_{0}\right]+q_{I I I}\left[\Phi-\Phi_{0}\right] \geq 0$. For $k=1, \ldots, \infty$ we get

$$
\begin{aligned}
& a_{k}^{\prime \prime}(x)-\frac{\pi^{2}}{d^{2}} k(k+1) a_{k}(x)=0 \\
& b_{k}^{\prime \prime}(x)-\frac{\pi^{2}}{d^{2}} k(k+1) b_{k}(x)=0
\end{aligned}
$$

and the square integrable solutions of these equations are

$$
\begin{aligned}
& a_{k}(x)=A_{k} \exp \left(\frac{\pi}{d} \sqrt{k(k+1)}(x+\delta)\right) \\
& b_{k}(x)=B_{k} \exp \left(\frac{\pi}{d} \sqrt{k(k+1)}(\delta-x)\right) .
\end{aligned}
$$

Putting these results to (3.16) and (3.17) we have

$$
q_{I}[\Phi]+q_{I I I}[\Phi] \geq \frac{\pi}{d} \sum_{k=1}^{\infty} \sqrt{k(k+1)}\left(\left|A_{k}\right|^{2}+\left|B_{k}\right|^{2}\right) .
$$


Let us turn our attention to the functional $q_{I I}$. First we estimate the value $\|\Phi\|_{L^{2}\left(\Omega_{I I}\right)}^{2}$. Denoting $\Phi_{-}(y)=\Phi(-\delta, y)$ and $\Phi_{+}(y)=\Phi(\delta, y)$ we can write

$$
\begin{aligned}
\|\Phi\|_{L^{2}\left(\Omega_{I I}\right)}^{2} & =\int_{0}^{d} \int_{-\delta}^{\delta}\left|\Phi_{-}(y)+\int_{-\delta}^{x} \frac{\partial \Phi}{\partial x}(z, y) \mathrm{d} z\right|\left|\int_{x}^{\delta} \frac{\partial \Phi}{\partial x}(z, y)-\Phi_{+}(y) \mathrm{d} z\right| \mathrm{d} x \mathrm{~d} y \leq \\
& \leq \int_{0}^{d} \int_{-\delta}^{\delta}\left(\left|\Phi_{-}(y)\right|+\int_{-\delta}^{\delta}\left|\frac{\partial \Phi}{\partial x}(z, y)\right| \mathrm{d} z\right)\left(\left|\Phi_{+}(y)\right|+\int_{-\delta}^{\delta}\left|\frac{\partial \Phi}{\partial x}(z, y)\right| \mathrm{d} z\right) \mathrm{d} x \mathrm{~d} y= \\
& =2 \delta \int_{0}^{d}\left(\left|\Phi_{-}(y)\right|\left|\Phi_{+}(y)\right|+\left(\left|\Phi_{-}(y)\right|+\left|\Phi_{+}(y)\right|\right) \int_{-\delta}^{\delta}\left|\frac{\partial \Phi}{\partial x}(z, y)\right| \mathrm{d} z+\right. \\
& \left.+\left(\int_{-\delta}^{\delta}\left|\frac{\partial \Phi}{\partial x}(z, y)\right| \mathrm{d} z\right)^{2}\right) \mathrm{d} y \leq 2 \delta\left(\left\|\Phi_{-}\right\|_{L^{2}(0, d)}\left\|\Phi_{+}\right\|_{L^{2}(0, d)}+\right. \\
& +\left\|\left|\Phi_{-}\right|+\left|\Phi_{+}\right|\right\|_{L^{2}(0, d)} \sqrt{\left.\int_{0}^{d}\left(\int_{-\delta}^{\delta}\left|\frac{\partial \Phi}{\partial x}(z, y)\right| \mathrm{d} z\right)^{2} \mathrm{~d} y+2 \delta\left\|\frac{\partial \Phi}{\partial x}\right\|_{L^{2}\left(\Omega_{I I}\right)}^{2}\right) \leq} \\
& \leq 2 \delta\left(\left\|\Phi_{-}\right\|_{L^{2}(0, d)}\left\|\Phi_{+}\right\|_{L^{2}(0, d)}+\sqrt{2 \delta}\left\|\left|\Phi_{-}\right|+\left|\Phi_{+}\right|\right\|_{L^{2}(0, d)}\left\|\frac{\partial \Phi}{\partial x}\right\|_{L^{2}\left(\Omega_{I I}\right)}+\right. \\
& \left.+2 \delta\left\|\frac{\partial \Phi}{\partial x}\right\|_{L^{2}\left(\Omega_{I I}\right)}^{2}\right),
\end{aligned}
$$

where we used the fact that $\Phi \in C^{2}\left(\Omega_{I I}\right)$ and the Schwarz inequality. Denoting $\kappa=\lambda \pi$, we use this estimate in the following

$$
\begin{aligned}
q_{I I}[\Phi] & =\left\|\frac{\partial \Phi}{\partial x}\right\|_{L^{2}\left(\Omega_{I I}\right)}^{2}+\left\|\frac{\partial \Phi}{\partial y}\right\|_{L^{2}\left(\Omega_{I I}\right)}^{2}-\frac{\pi^{2}}{4 d^{2}}\|\Phi\|_{L^{2}\left(\Omega_{I I}\right)}^{2} \geq \\
& \geq\left\|\frac{\partial \Phi}{\partial x}\right\|_{L^{2}\left(\Omega_{I I}\right)}^{2}\left(1-\kappa^{2}\right)-\sqrt{2 \delta} \frac{\kappa \pi}{2 d}\left\|\left|\Phi_{-}\right|+\left|\Phi_{+}\right|\right\|_{L^{2}(0, d)}\left\|\frac{\partial \Phi}{\partial x}\right\|_{L^{2}\left(\Omega_{I I}\right)}- \\
& -\frac{\kappa \pi}{2 d}\left\|\Phi_{-}\right\|_{L^{2}(0, d)}\left\|\Phi_{+}\right\|_{L^{2}(0, d)} .
\end{aligned}
$$

Let us assume that there exists $\Phi \in \mathcal{Q}(\Omega)$ such that $q[\Phi]<0$. Taking into consideration (3.20) we conclude that $q_{I I}[\Phi]<0$ for considered $\Phi \in \mathcal{Q}$. Then also

$\left\|\frac{\partial \Phi}{\partial x}\right\|_{L^{2}\left(\Omega_{I I}\right)}^{2}\left(1-\kappa^{2}\right)-\sqrt{2 \delta} \frac{\kappa \pi}{2 d}\left\|\left|\Phi_{-}\right|+\left|\Phi_{+}\right|\right\|_{L^{2}(0, d)}\left\|\frac{\partial \Phi}{\partial x}\right\|_{L^{2}\left(\Omega_{I I}\right)}-\frac{\kappa \pi}{2 d}\left\|\Phi_{-}\right\|_{L^{2}(0, d)}\left\|\Phi_{+}\right\|_{L^{2}(0, d)}<0$.

This inequality holds obviously for $\kappa \geq 1$. Let us solve this inequality for $\kappa<1$. Then using the triangle inequality together with the inequality

$$
2\left\|\Phi_{-}\right\|_{L^{2}(0, d)}\left\|\Phi_{+}\right\|_{L^{2}(0, d)} \leq \frac{1}{2}\left(\left\|\Phi_{-}\right\|_{L^{2}(0, d)}+\left\|\Phi_{+}\right\|_{L^{2}(0, d)}\right)^{2}
$$

we get

$$
\left\|\frac{\partial \Phi}{\partial x}\right\|_{L^{2}\left(\Omega_{I I}\right)}<\frac{\kappa}{2 \sqrt{2 \delta}(1-\kappa)}\left(\left\|\Phi_{-}\right\|_{L^{2}(0, d)}+\left\|\Phi_{+}\right\|_{L^{2}(0, d)}\right) .
$$

We use this result in the following estimate

$$
\begin{aligned}
\left\|\Phi_{+}-\Phi_{-}\right\|_{L^{2}(0, d)} & =\sqrt{\int_{0}^{d}\left|\int_{-\delta}^{\delta} \frac{\partial \Phi}{\partial x} \mathrm{~d} x\right|^{2} \mathrm{~d} y} \leq \sqrt{2 \delta}\left\|\frac{\partial \Phi}{\partial x}\right\|_{L^{2}\left(\Omega_{I I}\right)}< \\
& <\frac{\kappa}{2(1-\kappa)}\left(\left\|\Phi_{-}\right\|_{L^{2}(0, d)}+\left\|\Phi_{+}\right\|_{L^{2}(0, d)}\right) .
\end{aligned}
$$


Now we use estimates $(3.20),(3.21)$ together with inequality (3.22), the triangle inequality and

$$
\left(\left\|\Phi_{-}\right\|_{L^{2}(0, d)}+\left\|\Phi_{+}\right\|_{L^{2}(0, d)}\right)^{2} \leq 2\left(\left\|\Phi_{-}\right\|_{L^{2}(0, d)}^{2}+\left\|\Phi_{+}\right\|_{L^{2}(0, d)}^{2}\right)
$$

to estimate whole functional $q[\Phi]$. Denoting $A_{0}=a_{0}(-\delta), B_{0}=b_{0}(\delta)$ we obtain

$$
\begin{aligned}
q[\Phi] & \geq \frac{\pi}{d} \sum_{k=1}^{\infty} \sqrt{k(k+1)}\left(\left|A_{k}\right|^{2}+\left|B_{k}\right|^{2}\right)+\left\|\frac{\partial \Phi}{\partial x}\right\|_{L^{2}\left(\Omega_{I I}\right)}^{2}\left(1-\kappa^{2}\right)- \\
& -\sqrt{2 \delta} \frac{\kappa \pi}{2 d}\left\|\left|\Phi_{-}\right|+\left|\Phi_{+}\right|\right\|_{L^{2}(0, d)}\left\|\frac{\partial \Phi}{\partial x}\right\|_{L^{2}\left(\Omega_{I I}\right)}-\frac{\kappa \pi}{2 d}\left\|\Phi_{-}\right\|_{L^{2}(0, d)}\left\|\Phi_{+}\right\|_{L^{2}(0, d)} \geq \\
& \geq \frac{\pi \sqrt{2}}{d} \sum_{k=1}^{\infty}\left(\left|A_{k}\right|^{2}+\left|B_{k}\right|^{2}\right)+ \\
& +\left(1-\kappa^{2}\right)\left(\left\|\frac{\partial \Phi}{\partial x}\right\|_{L^{2}\left(\Omega_{I I}\right)}-\sqrt{2 \delta} \frac{\kappa \pi}{4 d} \frac{\left\|\left|\Phi_{-}\right|+\left|\Phi_{+}\right|\right\|_{L^{2}(0, d)}}{1-\kappa^{2}}\right)^{2}- \\
& -\frac{\kappa^{3} \pi}{8 d} \frac{\left\|\left|\Phi_{-}\right|+\left|\Phi_{+}\right|\right\|_{L^{2}(0, d)}^{2}-\frac{\kappa \pi}{4 d}\left(\left\|\Phi_{-}\right\|_{L^{2}(0, d)}^{2}+\left\|\Phi_{+}\right\|_{L^{2}(0, d)}^{2}\right) \geq}{\left(1-\kappa^{2}\right)} \\
& \geq \frac{\pi \sqrt{2}}{d} \sum_{k=1}^{\infty}\left(\left|A_{k}\right|^{2}+\left|B_{k}\right|^{2}\right)-\frac{\kappa \pi}{4 d\left(1-\kappa^{2}\right)} \sum_{k=0}^{\infty}\left(\left|A_{k}\right|^{2}+\left|B_{k}\right|^{2}\right),
\end{aligned}
$$

where we used also (3.14) and (3.15) together with (3.18) and (3.19). We assumed $q[\Phi]<0$, thus the same is true for the last expression in (3.26) and so we obtain for $\kappa<\frac{\sqrt{129}-1}{8 \sqrt{2}} \doteq 0.92$

$$
\sum_{k=1}^{\infty}\left(\left|A_{k}\right|^{2}+\left|B_{k}\right|^{2}\right)<\frac{\kappa}{4 \sqrt{2}\left(1-\kappa^{2}\right)-\kappa}\left(\left|A_{0}\right|^{2}+\left|B_{0}\right|^{2}\right) .
$$

Now we need estimates (3.25), (3.24) and (3.27) together with known inequalities for nonnegative numbers

$$
\begin{aligned}
2 a b & \leq a^{2}+b^{2} \\
(a+b+c)^{2} & \leq 3\left(a^{2}+b^{2}+c^{2}\right)
\end{aligned}
$$

and the triangle inequality to do the last step of the proof. First notice, that

$$
\frac{2}{d}\left\|A_{0} \sin \frac{\pi y}{2 d}-B_{0} \cos \frac{\pi y}{2 d}\right\|_{L^{2}(0, d)}^{2}=\left|A_{0}\right|^{2}+\left|B_{0}\right|^{2}-\frac{4}{\pi} \Re\left(A_{0} B_{0}\right) \geq\left(1-\frac{2}{\pi}\right)\left(\left|A_{0}\right|^{2}+\left|B_{0}\right|^{2}\right) .
$$


On the other hand

$$
\begin{aligned}
& \frac{2}{d}\left\|A_{0} \sin \frac{\pi y}{2 d}-B_{0} \cos \frac{\pi y}{2 d}\right\|_{L^{2}(0, d)}^{2}= \\
= & \left\|\Phi_{-}-\Phi_{+}-\sqrt{\frac{2}{d}} \sum_{k=1}^{\infty}\left(A_{k} \sin \frac{(2 k+1) \pi y}{2 d}-B_{k} \cos \frac{(2 k+1) \pi y}{2 d}\right)\right\|_{L^{2}(0, d)}^{2} \leq \\
\leq & \left(\left\|\Phi_{-}-\Phi_{+}\right\|_{L^{2}(0, d)}+\sqrt{\sum_{k=1}^{\infty}\left|A_{k}\right|^{2}}+\sqrt{\sum_{k=1}^{\infty}\left|B_{k}\right|^{2}}\right)^{2} \leq \\
\leq & \left(\frac{\kappa}{2(1-\kappa)}\left(\left\|\Phi_{-}\right\|_{L^{2}(0, d)}+\left\|\Phi_{+}\right\|_{L^{2}(0, d)}\right)+\sqrt{\sum_{k=1}^{\infty}\left|A_{k}\right|^{2}}+\sqrt{\sum_{k=1}^{\infty}\left|B_{k}\right|^{2}}\right)^{2}< \\
< & 3\left(\frac{\kappa^{2}}{2(1-\kappa)^{2}} \sum_{k=0}^{\infty}\left(\left|A_{k}\right|^{2}+\left|B_{k}\right|^{2}\right)+\sum_{k=1}^{\infty}\left(\left|A_{k}\right|^{2}+\left|B_{k}\right|^{2}\right)\right)< \\
< & 3 \frac{2 \sqrt{2}(1+\kappa) \kappa^{2}+\kappa(1-\kappa)}{(1-\kappa)\left(4 \sqrt{2}\left(1-\kappa^{2}\right)-\kappa\right)}\left(\left|A_{0}\right|^{2}+\left|B_{0}\right|^{2}\right) .
\end{aligned}
$$

We compare this inequality with (3.28) and we have

$$
1-\frac{2}{\pi}<3 \kappa \frac{2 \sqrt{2}(1+\kappa) \kappa+1-\kappa}{(1-\kappa)\left(4 \sqrt{2}\left(1-\kappa^{2}\right)-\kappa\right)} .
$$

We recall, that we investigate this inequality for $0<\kappa<\frac{\sqrt{129}-1}{8 \sqrt{2}}$. The right hand side of (3.29) is a positive, continuous function of $\kappa$ on this interval and it goes to zero as $\kappa$ does. Hence there must exist a number $\kappa_{0}$ (and so $\Lambda_{1}$ ), such that this inequality does no hold for $\kappa \in\left(0, \kappa_{0}\right)$, resp. $\lambda \in\left(0, \Lambda_{1}\right)$. Thus $q[\Phi] \geq 0$ for all $\Phi \in \mathcal{Q}(\Omega)$ for these values of the parameter $\lambda$, which has been to prove.

Remark 2 We also know that the discrete eigenvalues emerge at the essential spectrum treshold and they are continuous functions of $\lambda$ in both our specific cases. The rigorous formulation and the proof of this statement are given in Appendix A.

\section{Numerical results}

We have solved the Schrödinger equation corresponding to our systems numerically. Since $\Omega$ consists of three rectangular regions, the easiest way to do that is the mode-matching method (see e.g. 四). The results are shown in Figures 2-5. On the Figures 4 and 5 we can notice that the first eigenvalue in the model A appears for $\Lambda_{0}>0$. Our numerical results indicate that $\Lambda_{0} \doteq 0.26$ in agreement with our analytical proofs. Solving numerically the equation $q_{I I}\left[\Phi_{\sigma}\right]=0$ with right hand side given by (3.13) we get the result $\Lambda_{2} \doteq 0.34$. In the same way from (3.29) we get after numerical computation $\Lambda_{1} \doteq 0.08$. Hence $\Lambda_{1}<\Lambda_{0}<\Lambda_{2}$ which we have expected.

\section{Acknowledgements}

The authors thank Professor Pavel Exner for discussions on the problem formulation and expected results. The work is supported by GA ASCR grant IAA 1048101. 


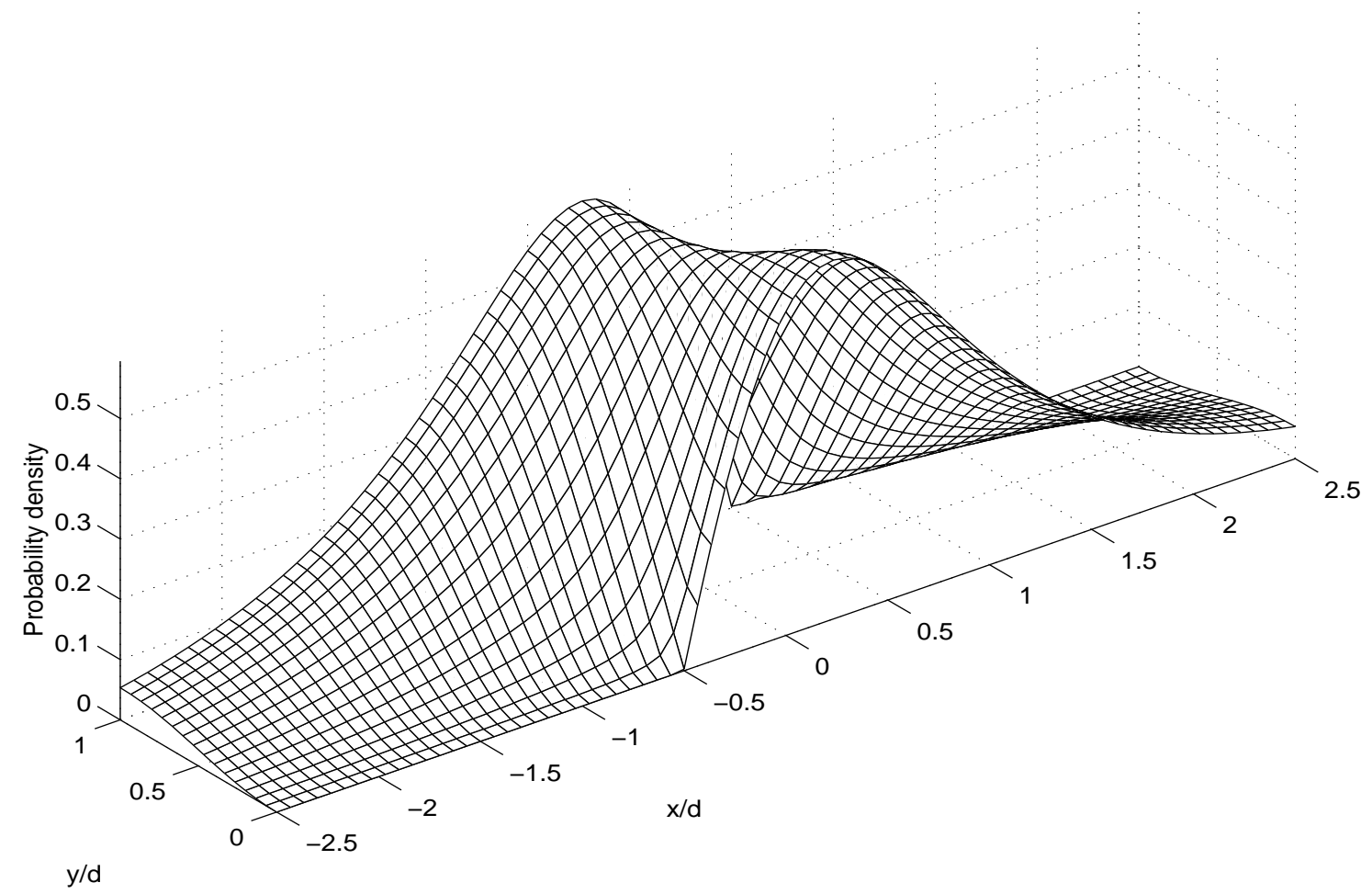

Figure 2: Probability density $|\psi|^{2}$ (in units of $d^{-2}$ ) for the bound state in model $\mathrm{A}, \lambda=\frac{1}{2}$, coordinates $x, y$ in the units of $d$. Only one discrete bound state exists here.

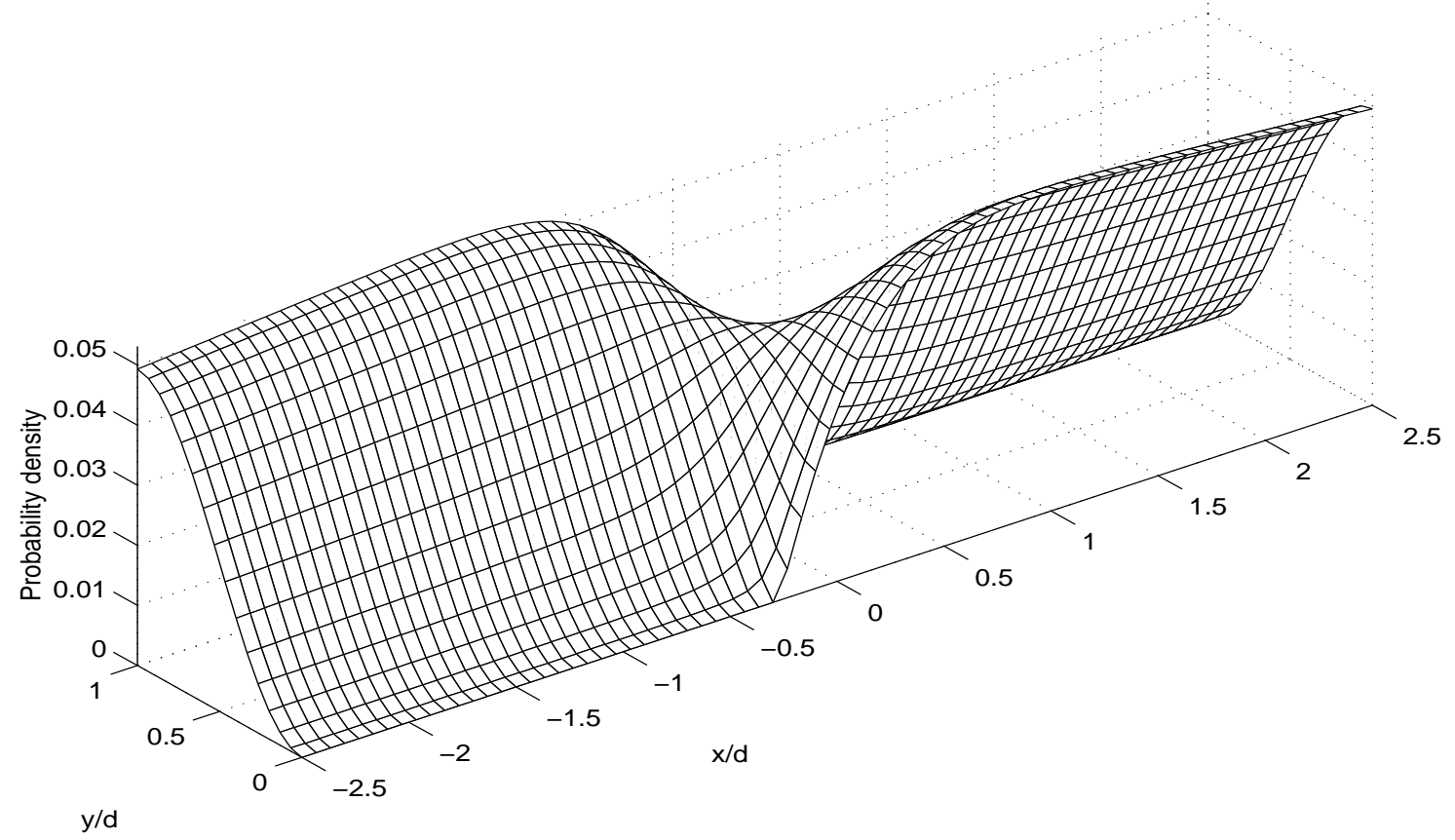

Figure 3: The same as in Fig.2 but $\lambda=0.27$. It is close to the treshold for bound state appearance. 


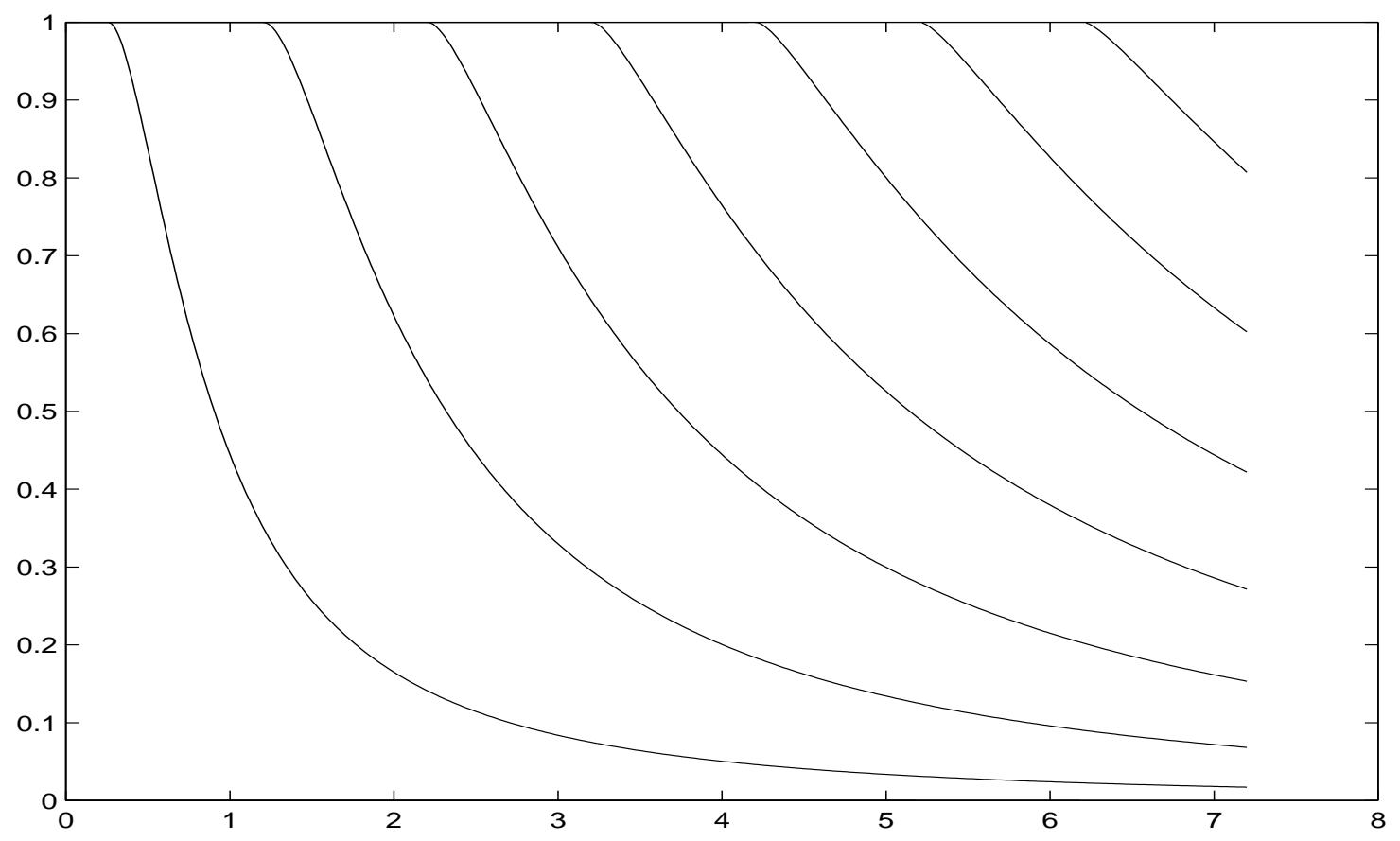

Figure 4: Eigenvalues (in the units of $\mu=\frac{\pi^{2}}{4 d^{2}}$ ) for the model A in dependence on $\lambda$.

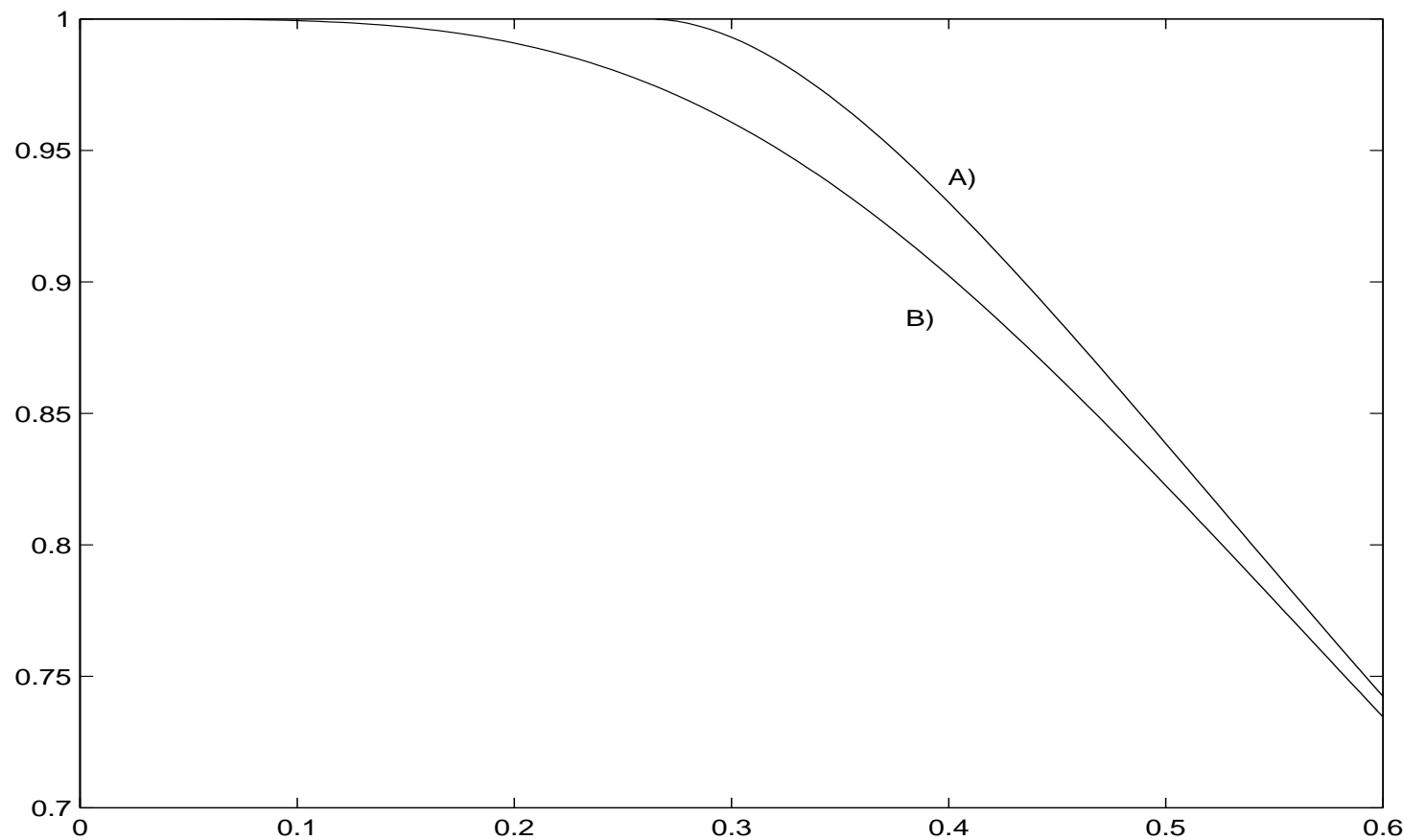

Figure 5: First eigenvalues for the model A and model B in dependence of $\lambda$. While in model $\mathrm{B}$ eigenvalue exists for any $\lambda>0$ in model $\mathrm{A}$ appears at $\Lambda>\Lambda_{0}$ (numerical calculations indicate $\left.\Lambda_{0} \doteq 0.26\right)$. 


\section{Appendix A: The continuity of the eigenvalues}

First we define some notations for purposes of this appendix. Let $\lambda>0$ then

$$
Q(\lambda)=\left\{f \in H^{1}(\Omega) \mid f\lceil\mathcal{D}(\lambda)=0\},\right.
$$

where

$$
\begin{aligned}
\mathcal{D}(\lambda) & =\{\langle x, 0\rangle \mid x<-\lambda d\} \cup\{\langle x, d\rangle \mid x>\lambda d\} \\
\text { or } & \\
\mathcal{D}(\lambda) & =\{\langle x, d\rangle \mid(x<-\lambda d) \vee(x>\lambda d)\}
\end{aligned}
$$

So we have stressed only the dependence of the form domain on the parameter $\lambda$. We define

$$
\begin{aligned}
& U\left(\varphi_{1}, \ldots, \varphi_{m} ; \lambda\right)= \inf _{\substack{\psi \in Q(\lambda), \psi \neq 0 \\
\psi \perp \varphi_{1}, \ldots, \varphi_{m}}} \frac{\|\nabla \psi\|_{L^{2}(\Omega)}^{2}}{\|\psi\|_{L^{2}(\Omega)}^{2}} \\
& \mu_{n}(\lambda)=\sup _{\varphi_{1}, \ldots, \varphi_{n-1} \in L^{2}(\Omega)} U\left(\varphi_{1}, \ldots, \varphi_{n-1} ; \lambda\right) .
\end{aligned}
$$

From minimax principle [11, we know that for every $n=1,2, \ldots, \mu_{n}(\lambda)$ is either the $n$-th eigenvalue of the operator (counting multiplicity) or the bottom of its essential spectrum. The aim of this appendix is to show that $\mu_{n}(\lambda)$ are continuous functions in $(0, \infty)$.

Lemma 1 Functions $\mu_{n}: \lambda \mapsto \mu_{n}(\lambda)$ are nonincreasing, finite and continuous in $(0, \infty)$ for every $n=1,2, \ldots$.

Proof: We know from the minimax principle and the Dirichlet-Neumann bracketing 11 that $0 \leq \mu_{n}(\lambda) \leq \frac{\pi^{2}}{4 d^{2}}$ for all $\lambda>0$ and $n=1,2, \ldots$ Let $\lambda_{1}>\lambda_{2}$. Then $Q\left(\lambda_{2}\right) \subset Q\left(\lambda_{1}\right)$ and so for any $m=0,1, \ldots$ and any $m$-tuple $\varphi_{1}, \ldots, \varphi_{m}$

$$
U\left(\varphi_{1}, \ldots, \varphi_{m} ; \lambda_{1}\right) \leq U\left(\varphi_{1}, \ldots, \varphi_{m} ; \lambda_{2}\right) \leq \mu_{m+1}\left(\lambda_{2}\right) .
$$

Because this inequalities hold for every $m$-tuple $\varphi_{1}, \ldots, \varphi_{m}$ from our Hilbert space they must be fulfilled even for supremum over these $m$-tuples. Hence

$$
\mu_{m+1}\left(\lambda_{1}\right) \leq \mu_{m+1}\left(\lambda_{2}\right)
$$

for every $m=0,1, \ldots$ and $\mu_{m+1}$ are thus nonincreasing.

For any $\varrho>1$ we define $\varphi^{(\varrho)}(x, y)=\varphi(\varrho x, y)$. The equivalences $\varphi \in Q(\lambda) \Leftrightarrow \varphi^{(\varrho)} \in Q\left(\frac{\lambda}{\varrho}\right)$ and $\varphi \perp \psi \Leftrightarrow \varphi^{(\varrho)} \perp \psi^{(\varrho)}$ are obvious. Moreover

$$
\frac{\left\|\nabla \varphi^{(\varrho)}\right\|_{L^{2}(\Omega)}^{2}}{\left\|\varphi^{(\varrho)}\right\|_{L^{2}(\Omega)}^{2}}=\frac{\varrho^{2}\left\|\frac{\partial \varphi}{\partial x}\right\|_{L^{2}(\Omega)}^{2}+\left\|\frac{\partial \varphi}{\partial y}\right\|_{L^{2}(\Omega)}^{2}}{\|\varphi\|_{L^{2}(\Omega)}^{2}} \leq \varrho^{2} \frac{\|\nabla \varphi\|_{L^{2}(\Omega)}^{2}}{\|\varphi\|_{L^{2}(\Omega)}^{2}} .
$$

Let integer $m \geq 0, \lambda>0$ and $\varphi_{1}, \ldots, \varphi_{m} \in L^{2}(\Omega)$ are chosen arbitrarily. Then for any $\varphi \in Q(\lambda)$ such that $\varphi \perp \varphi_{1}, \ldots, \varphi_{m}$ we know $\varphi_{1}^{(\varrho)}, \ldots, \varphi_{m}^{(\varrho)} \in L^{2}(\Omega), \varphi^{(\varrho)} \in Q\left(\frac{\lambda}{\varrho}\right)$ and $\varphi^{(\varrho)} \perp \varphi_{1}^{(\varrho)}, \ldots, \varphi_{m}^{(\varrho)}$. Then using (42) we get

$$
U\left(\varphi_{1}^{(\varrho)}, \ldots, \varphi_{m}^{(\varrho)} ; \frac{\lambda}{\varrho}\right) \leq \frac{\left\|\nabla \varphi^{(\varrho)}\right\|_{L^{2}(\Omega)}^{2}}{\left\|\varphi^{(\varrho)}\right\|_{L^{2}(\Omega)}^{2}} \leq \varrho^{2} \frac{\|\nabla \varphi\|_{L^{2}(\Omega)}^{2}}{\|\varphi\|_{L^{2}(\Omega)}^{2}} .
$$


Because these inequalities hold for arbitrary $m$-tuple $\varphi_{1}, \ldots \varphi_{m}$ it must hold even for the infimum of $\frac{\|\nabla \varphi\|_{L^{2}(\Omega)}^{2}}{\|\varphi\|_{L^{2}(\Omega)}^{2}}$ and hence

$$
U\left(\varphi_{1}^{(\varrho)}, \ldots, \varphi_{m}^{(\varrho)} ; \frac{\lambda}{\varrho}\right) \leq \varrho^{2} U\left(\varphi_{1}, \ldots, \varphi_{m} ; \lambda\right) \leq \varrho^{2} \mu_{m+1}(\lambda)
$$

Passing again to the supremum over all $m$-tuples $\varphi_{1}^{(\varrho)}, \ldots, \varphi_{m}^{(\varrho)}$ and taking into account (A1) we obtain

$$
\mu_{m+1}(\lambda) \leq \mu_{m+1}\left(\frac{\lambda}{\varrho}\right) \leq \varrho^{2} \mu_{m+1}(\lambda)
$$

because $\lambda>\frac{\lambda}{\varrho}$. From the second inequality of (A3) and from (A1) we get

$$
\frac{1}{\varrho^{2}} \mu_{m+1}(\lambda) \leq \mu_{m+1}(\lambda \varrho) \leq \mu_{m+1}(\lambda)
$$

using $\lambda \varrho>\lambda$. We recall that these inequalities hold for any $m \in\{0,1, \ldots\}$.

For any $0<\lambda^{\prime}<\lambda$ we set $\varrho=\frac{\lambda}{\lambda^{\prime}}$ and we put it to (A3)

$$
\mu_{m+1}(\lambda) \leq \mu_{m+1}\left(\lambda^{\prime}\right) \leq\left(\frac{\lambda}{\lambda^{\prime}}\right)^{2} \mu_{m+1}(\lambda)
$$

We see that $\lim _{\lambda^{\prime} \rightarrow \lambda^{-}} \mu_{m+1}\left(\lambda^{\prime}\right)=\mu_{m+1}(\lambda)$. We repeat the same procedure for $0<\lambda<\lambda^{\prime}$ using the inequalities (A4) instead of (A3) and we get $\lim _{\lambda^{\prime} \rightarrow \lambda^{+}} \mu_{m+1}\left(\lambda^{\prime}\right)=\mu_{m+1}(\lambda)$, which finishes the proof.

\section{Appendix B: The dense set in $Q^{A}\left(q_{0}\right)$}

The goal of the appendix is to prove the following lemma.

Lemma 2 The set $\tilde{Q}(\Omega)=\left\{\psi \in C^{\infty}(\bar{\Omega}) \mid \psi=\tilde{\psi} \uparrow \Omega, \tilde{\psi} \in C_{0}^{\infty}\left(\mathbb{R}^{2}\right), \psi \uparrow \mathcal{D}=0\right\}$ is dense in the set $Q^{A}\left(q_{0}\right)$ with respect to the $H^{1}(\Omega)$ norm.

Proof: Let $\varphi \in Q^{A}\left(q_{0}\right)$. Then for every $\varepsilon>0$ we can find the function $\varphi_{1}^{(\varepsilon)}$ from the set $\left\{\psi \in C^{\infty}(\bar{\Omega}) \mid \psi=\tilde{\psi}\left\lceil\Omega, \tilde{\psi} \in C_{0}^{\infty}\left(\mathbb{R}^{2}\right)\right\}\right.$ such that $\left\|\varphi-\varphi_{1}^{(\varepsilon)}\right\|_{H^{1}(\Omega)}<\varepsilon$ (see e.g. [9], Theorem 3.18). Due to the Theorem 5.22 in [9] we can also write $\left\|\left(\varphi-\varphi_{1}^{(\varepsilon)}\right) \backslash \mathcal{D}\right\|_{L^{2}(\mathcal{D})}<\sqrt{C} \varepsilon$, where $C$ is a constant. Let $\gamma$ be the function from the class $C^{\infty}(\mathbb{R})$ with the following properties: $0 \leq \gamma(t) \leq 1$ for $t \in \mathbb{R}, \gamma(t)=0$ for $t \leq \frac{1}{2}, \gamma(t)=1$ for $t \geq 1$ and $\left|\frac{d \gamma(t)}{d t}\right| \leq \frac{\Gamma}{5}$ for some constant $\Gamma$ and every $t \in \mathbb{R}$. For $\langle x, y\rangle \in \Omega$ and $\varepsilon<d / 2$ we define

$$
\begin{aligned}
& \omega_{\varepsilon}^{(1)}(x, y)=\left\{\begin{array}{ll}
\gamma\left(\frac{4(d-y)}{\varepsilon}\right) \gamma\left(\frac{\sqrt{(x-\delta)^{2}+(y-d)^{2}}}{\varepsilon}\right) & \text { for } \quad x \geq \delta \\
\gamma\left(\frac{\sqrt{(x-\delta)^{2}+(y-d)^{2}}}{\varepsilon}\right) & \text { for } \quad x \leq \delta
\end{array},\right. \\
& \omega_{\varepsilon}^{(2)}(x, y)= \begin{cases}\gamma\left(\frac{4 y}{\varepsilon}\right) \gamma\left(\frac{\sqrt{(x+\delta)^{2}+y^{2}}}{\varepsilon}\right) & \text { for } \quad x \leq-\delta \\
\gamma\left(\frac{\sqrt{(x+\delta)^{2}+y^{2}}}{\varepsilon}\right) & \text { for } \quad x \geq-\delta\end{cases}
\end{aligned}
$$


and $\omega_{\varepsilon}=\omega_{\varepsilon}^{(1)} \omega_{\varepsilon}^{(2)}$. Obviously $\varphi_{2}^{(\varepsilon)}=\varphi_{1}^{(\varepsilon)} \omega_{\varepsilon} \in \tilde{Q}(\Omega)$. Let we denote

$$
\begin{aligned}
& \Omega_{\varepsilon}=\{\langle x, y\rangle \in \Omega \mid \operatorname{dist}(\langle x, y\rangle, \mathcal{D})<\varepsilon\} \\
& \Omega_{\varepsilon}^{1}=((\delta, \infty) \times(d-\varepsilon, d)) \cup((-\infty,-\delta) \times(0, \varepsilon)) \\
& \Omega_{\varepsilon}^{2}=((\delta-\varepsilon, \delta) \times(d-\varepsilon, d)) \cup((-\delta,-\delta+\varepsilon) \times(0, \varepsilon)) \\
& \Omega_{\varepsilon}^{3}=((\delta-\varepsilon, \delta+\varepsilon) \times(d-\varepsilon, d)) \cup((-\delta-\varepsilon,-\delta+\varepsilon) \times(0, \varepsilon)) .
\end{aligned}
$$

Now we are going to estimate $\left\|\varphi_{2}^{(\varepsilon)}-\varphi\right\|_{H^{1}(\Omega)}^{2}$.

$$
\begin{aligned}
\left\|\varphi_{2}^{(\varepsilon)}-\varphi\right\|_{H^{1}(\Omega)}^{2} & =\left\|\left(\varphi_{1}^{(\varepsilon)}-\varphi\right) \omega_{\varepsilon}+\varphi\left(\omega_{\varepsilon}-1\right)\right\|_{L^{2}(\Omega)}^{2}+ \\
& +\left\|\nabla\left(\varphi_{1}^{(\varepsilon)}-\varphi\right) \omega_{\varepsilon}+\nabla \varphi\left(\omega_{\varepsilon}-1\right)+\varphi_{1}^{(\varepsilon)} \nabla \omega_{\varepsilon}\right\|_{L^{2}(\Omega)}^{2} \leq \\
& \leq 3\left(\left\|\varphi_{1}^{(\varepsilon)}-\varphi\right\|_{H^{1}(\Omega)}^{2}+\|\varphi\|_{H^{1}\left(\Omega_{\varepsilon}\right)}^{2}+\frac{\Gamma^{2}}{\varepsilon^{2}}\left\|\varphi_{1}^{(\varepsilon)}\right\|_{L^{2}\left(\Omega_{\varepsilon}\right)}^{2}\right)
\end{aligned}
$$

Now we continue by estimating of $\left\|\varphi_{1}^{(\varepsilon)}\right\|_{L^{2}\left(\Omega_{\varepsilon}\right)}^{2}$.

$$
\begin{aligned}
\left\|\varphi_{1}^{(\varepsilon)}\right\|_{L^{2}\left(\Omega_{\varepsilon}\right)}^{2} & \leq\left\|\varphi_{1}^{(\varepsilon)}\right\|_{L^{2}\left(\Omega_{\varepsilon}^{1}\right)}^{2}+\left\|\varphi_{1}^{(\varepsilon)}\right\|_{L^{2}\left(\Omega_{\varepsilon}^{2}\right)}^{2} \\
\left\|\varphi_{1}^{(\varepsilon)}\right\|_{L^{2}\left(\Omega_{\varepsilon}^{1}\right)}^{2} & =\int_{\delta}^{\infty} \int_{d-\varepsilon}^{d}\left|\varphi_{1}^{(\varepsilon)}(x, y)\right|^{2} \mathrm{~d} y \mathrm{~d} x+\int_{-\infty}^{-\delta} \int_{0}^{\varepsilon}\left|\varphi_{1}^{(\varepsilon)}(x, y)\right|^{2} \mathrm{~d} y \mathrm{~d} x
\end{aligned}
$$

Because the estimates of the second term in the previous expression will be analogous to ones of the first term we will not write the second integral, but only "second term" instead of it.

$$
\begin{aligned}
\left\|\varphi_{1}^{(\varepsilon)}\right\|_{L^{2}\left(\Omega_{\varepsilon}^{1}\right)}^{2} & =\int_{\delta}^{\infty} \int_{d-\varepsilon}^{d}\left|\varphi_{1}^{(\varepsilon)}(x, d)-\int_{y}^{d} \frac{\partial \varphi_{1}^{(\varepsilon)}}{\partial t}(x, t) \mathrm{d} t\right|^{2} \mathrm{~d} y \mathrm{~d} x+\text { second term } \leq \\
& \leq 2 \int_{\delta}^{\infty} \int_{d-\varepsilon}^{d}\left(\left|\varphi_{1}^{(\varepsilon)}(x, d)\right|^{2}+\varepsilon \int_{d-\varepsilon}^{d}\left|\frac{\partial \varphi_{1}^{(\varepsilon)}}{\partial t}(x, t)\right|^{2} \mathrm{~d} t\right) \mathrm{d} y \mathrm{~d} x+\text { second term }= \\
& =2\left(\varepsilon\left\|\varphi_{1}^{(\varepsilon)} \mid \mathcal{D}\right\|_{L^{2}(\mathcal{D})}^{2}+\varepsilon^{2}\left\|\frac{\partial \varphi_{1}^{(\varepsilon)}}{\partial y}\right\|_{L^{2}\left(\Omega_{\varepsilon}^{1}\right)}^{2}\right) \leq 2 \varepsilon^{2}\left(C \varepsilon+\left\|\varphi_{1}^{(\varepsilon)}\right\|_{H^{1}\left(\Omega_{\varepsilon}^{1}\right)}^{2}\right) \leq \\
& \leq 2 \varepsilon^{2}\left(C \varepsilon+2\left(\left\|\varphi_{1}^{(\varepsilon)}-\varphi\right\|_{H^{1}(\Omega)}^{2}+\|\varphi\|_{H^{1}\left(\Omega_{\varepsilon}^{1}\right)}^{2}\right)\right) \leq 2 \varepsilon^{2}\left(C \varepsilon+2 \varepsilon^{2}+2\|\varphi\|_{H^{1}\left(\Omega_{\varepsilon}^{1}\right)}^{2}\right)
\end{aligned}
$$

For any $z \in(\delta, \delta+\varepsilon)$ we calculate

$$
\begin{aligned}
\left\|\varphi_{1}^{(\varepsilon)}\right\|_{L^{2}\left(\Omega_{\varepsilon}^{2}\right)}^{2} & =\int_{\delta-\varepsilon}^{\delta} \int_{d-\varepsilon}^{d}\left|\int_{x}^{z} \frac{\partial \varphi_{1}^{(\varepsilon)}}{\partial t}(t, y) \mathrm{d} t+\int_{y}^{d} \frac{\partial \varphi_{1}^{(\varepsilon)}}{\partial s}(z, s) \mathrm{d} s-\varphi_{1}^{(\varepsilon)}(z, d)\right|^{2} \mathrm{~d} y \mathrm{~d} x+\text { s.t. } \leq \\
& \leq 3 \int_{\delta-\varepsilon}^{\delta} \int_{d-\varepsilon}^{d}\left(2 \varepsilon \int_{\delta-\varepsilon}^{\delta+\varepsilon}\left|\frac{\partial \varphi_{1}^{(\varepsilon)}}{\partial t}(t, y)\right|^{2} \mathrm{~d} t+\varepsilon \int_{d-\varepsilon}^{d}\left|\frac{\partial \varphi_{1}^{(\varepsilon)}}{\partial s}(z, s)\right|^{2} \mathrm{~d} s+\right. \\
& \left.+\left|\varphi_{1}^{(\varepsilon)}(z, d)\right|^{2}\right) \mathrm{d} y \mathrm{~d} x+\text { second term. }
\end{aligned}
$$

When we integrate the last inequality over $z$ from $\delta$ to $\delta+\varepsilon$ we obtain

$$
\varepsilon\left\|\varphi_{1}^{(\varepsilon)}\right\|_{L^{2}\left(\Omega_{\varepsilon}^{2}\right)}^{2} \leq 3\left(2 \varepsilon^{3}\left\|\frac{\partial \varphi_{1}^{(\varepsilon)}}{\partial x}\right\|_{L^{2}\left(\Omega_{\varepsilon}^{3}\right)}^{2}+\varepsilon^{3}\left\|\frac{\partial \varphi_{1}^{(\varepsilon)}}{\partial y}\right\|_{L^{2}\left(\Omega_{\varepsilon}^{3}\right)}^{2}+\varepsilon^{2} \| \varphi_{1}^{(\varepsilon)}\left\lceil\mathcal{D} \|_{L^{2}(\mathcal{D})}^{2}\right)\right.
$$


and so

$$
\left\|\varphi_{1}^{(\varepsilon)}\right\|_{L^{2}\left(\Omega_{\varepsilon}^{2}\right)}^{2} \leq 6 \varepsilon^{2}\left\|\varphi_{1}^{(\varepsilon)}\right\|_{H^{1}\left(\Omega_{\varepsilon}^{3}\right)}^{2}+3 C \varepsilon^{3} \leq 12 \varepsilon^{2}\left(\varepsilon^{2}+\|\varphi\|_{H^{1}\left(\Omega_{\varepsilon}^{3}\right)}^{2}\right)+3 C \varepsilon^{3} .
$$

We give both these estimates together and we get

$$
\left\|\varphi_{1}^{(\varepsilon)}\right\|_{L^{2}\left(\Omega_{\varepsilon}\right)}^{2} \leq 5 C \varepsilon^{3}+16 \varepsilon^{4}+16 \varepsilon^{2}\|\varphi\|_{H^{1}\left(\Omega_{\varepsilon}^{1} \cup \Omega_{\varepsilon}^{2}\right)}^{2}
$$

Hence

$$
\left\|\varphi_{2}^{(\varepsilon)}-\varphi\right\|_{H^{1}(\Omega)}^{2} \leq 3\left(\left(1+16 \Gamma^{2}\right)\left(\varepsilon^{2}+\|\varphi\|_{H^{1}\left(\Omega_{\varepsilon}^{1} \cup \Omega_{\varepsilon}^{2}\right)}^{2}\right)+5 C \Gamma^{2} \varepsilon\right) .
$$

Here $\lim _{\varepsilon \rightarrow 0^{+}}\|\varphi\|_{H^{1}\left(\Omega_{\varepsilon}^{1} \cup \Omega_{\varepsilon}^{2}\right)}^{2}=0$, e.g. by the dominated convergence. So $\left\|\varphi_{2}^{(\varepsilon)}-\varphi\right\|_{H^{1}(\Omega)}^{2}$ goes to zero as $\varepsilon$ does which has been to show.

Remark 3 Our set $\tilde{Q}$ is obviously a subset of the set $\mathcal{Q}$ defined in the proof of the Theorem 3. Hence $\mathcal{Q}$ is a dense set in $Q^{A}\left(q_{0}\right)$ as well.

\section{References}

[1] P. Exner, P. Šeba: "Bound states in curved waveguides", J. Math. Phys. 30, 2574-2580 (1989).

[2] J. Goldstone, R.L. Jaffe: "Bound states in twisting tubes", Phys. Rev. B45, 14100-14107 (1992).

[3] P. Duclos, P. Exner: "Curvature-induced bound states in quantum waveguides in two and three dimensions", Rev.Math.Phys. 7, 73-102(1995).

[4] P. Exner, P. Šeba, M. Tater, D. Vaněk: "Bound states and scattering in quantum waveguides coupled laterally through a boundary window", J. Math. Phys. 37, 4867-4887 (1996).

[5] I.Yu. Popov: "Asymptotics of Bound State for Laterally Coupled Waveguides", Rep. Math. Phys. 43, 427-437 (1999).

[6] N.E. Hurt: Mathematical physics of quantum wires and devices, Mathematics and its application, 506, Kluwer Academic Publishers, Dordrecht 2000.

[7] S.A. Nazarov, M. Specovius-Neugebauer: "Selfadjoint extensions of the Neumann Laplacian in domanis with cylindrical outlets", Commun. Math. Phys. 185, 689-707 (1997).

[8] E.B. Davies, L. Parnovski: "Trapped modes in acoustic waveguides", Q. J. Mech. Appl. Math. 51, 477-492(1998).

[9] R.A. Adams: Sobolev Spaces, Academic Press, New York 1975.

[10] J. Blank, P. Exner, M. Havlíček: Hilbert Space Operators in Quantum Physics, AIP, New York 1994.

[11] M. Reed, B. Simon: Methods of Modern Mathematical Physics, I. Functional Analysis, II. Fourier Analysis,Self-Adjointness, IV. Analysis of Operators, Academic Press, New York 1972, 1975, 1978.

[12] N. Dunford, J.T. Schwartz: Linear Operators, Part II: Spectral Theory, Self Adjoint Operators in Hilbert Space, Interscience Publishers, New York 1963.

[13] M.S. Birman, G.E. Skvortsov: "Square summability of the highest derivatives of the solution of the Dirichlet boundary value problem in a domain with a piecewise smooth boundary" (in Russian), Izvestia vysshikh uchebnykh zavedenii, Matematika 30, No. 5, $12-21$ (1962).

[14] E.B. Davies: Spectral Theory and Differential Operators, University Press, Cambridge 1995 . 